\title{
Systems Biomedicine of Rabies Delineates the Affected Signaling Pathways
}

\author{
Sadegh Azimzadeh Jamalkandi ${ }^{1 \dagger}$, Sayed-Hamidreza Mozhgani ${ }^{2 \dagger}$, \\ Hamid Gholami Pourbadie ${ }^{3}$, Mehdi Mirzaie ${ }^{4}$, Farshid Noorbakhsh ${ }^{5}$, Behrouz Vaziri ${ }^{6}$, \\ Alireza Gholami ${ }^{7 *}$, Naser Ansari-Pour ${ }^{8,9 *}$ and Mohieddin Jafari ${ }^{10 *}$ \\ ${ }^{1}$ Chemical Injuries Research Center, Baqiyatallah University of Medical Sciences, Tehran, Iran, ${ }^{2}$ Department of Virology, \\ School of Public Health, Tehran University of Medical Sciences, Tehran, Iran, ${ }^{3}$ Department of Physiology and Pharmacology, \\ Pasteur Institute of Iran, Tehran, Iran, ${ }^{4}$ Department of Applied Mathematics, Faculty of Mathematical Sciences, Tarbiat \\ Modares University, Tehran, Iran, ${ }^{5}$ Department of Immunology, School of Medicine, Tehran University of Medical Sciences, \\ Tehran, Iran, ${ }^{6}$ Protein Chemistry and Proteomics Unit, Medical Biotechnology Department, Biotechnology Research Center, \\ Pasteur Institute of Iran, Tehran, Iran, ${ }^{7}$ WHO Collaborating Center for Reference and Research on Rabies, Pasteur Institute of \\ Iran, Tehran, Iran, ${ }^{8}$ Faculty of New Sciences and Technology, University of Tehran, Tehran, Iran, ${ }^{9}$ Department of Genetics, \\ Evolution and Environment, UCL Genetics Institute, University College London, London, UK, ${ }^{10}$ Drug Design and \\ Bioinformatics Unit, Medical Biotechnology Department, Biotechnology Research Center, Pasteur Institute of Iran, Tehran, \\ Iran
}

Reviewed by: Takashi Irie

Hiroshima University, Japan Iman Tavassoly, Icahn School of Medicine at Mount

Sinai, USA

*Correspondence: Alireza Gholam a.gholami@pasteur.ac.ir

Naser Ansari-Pour n.ansaripour@ut.ac.ir Mohieddin Jafari m_jafari@pasteur.ac.ir; mjafari@ipm.ir;

www.jafarilab-pasteur.com

${ }^{\dagger}$ These authors have contributed equally to this work.

Specialty section: This article was submitted to Virology,

a section of the journal

Frontiers in Microbiology

Received: 10 August 2016 Accepted: 07 October 2016 Published: 07 November 2016

Citation:

Azimzadeh Jamalkandi $S$, Mozhgani S-H, Gholami Pourbadie H, Mirzaie $M$, Noorbakhsh F, Vaziri $B$,

Gholami A, Ansari-Pour N and Jafari M (2016) Systems Biomedicine of Rabies Delineates the Affected Signaling Pathways.

Front. Microbiol. 7:1688. doi: 10.3389/fmicb.2016.01688

The prototypical neurotropic virus, rabies, is a member of the Rhabdoviridae family that causes lethal encephalomyelitis. Although there have been a plethora of studies investigating the etiological mechanism of the rabies virus and many precautionary methods have been implemented to avert the disease outbreak over the last century, the disease has surprisingly no definite remedy at its late stages. The psychological symptoms and the underlying etiology, as well as the rare survival rate from rabies encephalitis, has still remained a mystery. We, therefore, undertook a systems biomedicine approach to identify the network of gene products implicated in rabies. This was done by meta-analyzing whole-transcriptome microarray datasets of the CNS infected by strain CVS-11, and integrating them with interactome data using computational and statistical methods. We first determined the differentially expressed genes (DEGs) in each study and horizontally integrated the results at the mRNA and microRNA levels separately. A total of 61 seed genes involved in signal propagation system were obtained by means of unifying mRNA and microRNA detected integrated DEGs. We then reconstructed a refined protein-protein interaction network (PPIN) of infected cells to elucidate the rabies-implicated signal transduction network (RISN). To validate our findings, we confirmed differential expression of randomly selected genes in the network using Real-time PCR. In conclusion, the identification of seed genes and their network neighborhood within the refined PPIN can be useful for demonstrating signaling pathways including interferon circumvent, toward proliferation and survival, and neuropathological clue, explaining the intricate underlying molecular neuropathology of rabies infection and thus rendered a molecular framework for predicting potential drug targets.

Keywords: rabies, systems biology, protein-protein interaction network, signaling network, microarray, real-time PCR 


\section{INTRODUCTION}

Growing evidence of inter-population and inter-individual variation in the attack rate and prognosis of specific infectious diseases suggest an underlying biological complexity. In fact, any perturbation in the densely organized inter-relationship of genetic and environmental factors may lead to this intricate behavior (Hunter, 2005). In particular, the strange survival pattern observed from fatal rabies infection of the central nervous system (CNS) introduces this infection as a complex disease (de Souza and Madhusudana, 2014).

The prototypical neurotropic virus, rabies, is a member of the Rhabdoviridae family that causes lethal encephalomyelitis (Sugiura et al., 2011). The viruses in this family are enveloped with a single stranded negative sense RNA genome. The genomic length of the rabies virus (RABV) is about $12 \mathrm{~kb}$ and encodes five proteins including nucleoprotein $(\mathrm{N})$, phosphoprotein $(\mathrm{P})$, matrix protein $(\mathrm{M})$, glycoprotein $(\mathrm{G})$, and a viral RNA polymerase (L) (Yousaf et al., 2012). This neglected virus leads to death once the symptoms develop and has a mortality rate of $1: 100,000$ to $1: 1000$ per year. The deceased intriguingly display no neural damage, neurohistopathological evidence, or induced severe immune response (Schnell et al., 2010). In an organized hijacking program, the virus travels from the muscle tissue to the nervous system, migrates to the spinal cord and freely covers certain parts of the brain (Schnell et al., 2010). The virus spreads centrifugally to other organs and subsequently to the next host. Although the host innate immune response including TLR, type 1 interferon, TNF alpha, and IL-6 are the first defense line against a viral infection, this virus easily propagates in the nervous system. This suggests that the RABV has a specific mechanism to suppress host innate immunity (Rupprecht, 1996; Ito et al., 2011; Gomme et al., 2012). Several laboratory strains of the RABV in addition to the wild types cause fatal acute encephalomyelitis associated with inflammation of the brain and spinal cord, leading to coma and death especially when the virus is injected intracerebrally in high dose (Meslin et al., 1996; Galelli et al., 2000; Baloul and Lafon, 2003; Baloul et al., 2004). In contrast to attenuated strains, wild type strains and CVS-11 do not induce histopathological changes indicative of apoptosis or necrosis in infected cells (Thoulouze et al., 1997, 2003a,b; Lay et al., 2003; Préhaud et al., 2003). Accordingly, despite over 100 years of controlling rabies by developing RABV vaccines and serotherapy, the precise neurological and immunological etiology as well as rare survival cases from rabies encephalitis still remains a mystery (Gomme et al., 2012; de Souza and Madhusudana, 2014).

After the emergence of omics technology, some studies have started to pave the way toward a better understanding of rabies fatal mechanism. Elucidating the essential biological processes involved in rabies progression has been based mainly on analyzing gene expression alterations. Zhao et al. reported expression profiling of mRNA and microRNA of rabies-infected cell (Zhao et al., 2011, 2012a,b, 2013). Suigiura et al. analyzed the gene expression profile of CNS tissue infected with CVS11 (Sugiura et al., 2011). Changes in gene expression were also studied in marked neurons infected with recombinant RABV expressing CRE-recombinase (Gomme et al., 2012). Numerous other studies have also analyzed gene expression profiling using transcriptomic or proteomic methods within diverse cellular models in different species (Wang et al., 2005, 2011; Dhingra et al., 2007; Fu et al., 2008; Zandi et al., 2009, 2013; Han et al., 2011; Thanomsridetchai et al., 2011; Vaziri et al., 2012; Farahtaj et al., 2013; Francischetti et al., 2013; Kluge et al., 2013; Silva et al., 2013; Venugopal et al., 2013; Kasempimolporn et al., 2014; Kammouni et al., 2015; Mehta et al., 2015).

To increase the reliability of results and generalizability of these independent but related studies, it is recommended to statistically combine such data, commonly known as data integration or meta-analysis (Ramasamy et al., 2008). Several studies have shown the benefits of meta-analysis in terms of both higher statistical power and precision in detecting differentially expressed genes (DEGs) in different complex traits including infectious disease (Song et al., 2014; Camacho-Cáceres et al., 2015; Sharma et al., 2015; Yin et al., 2015; Wang C.-Y. et al., 2015; Wang X. et al., 2015). Further, data integration approaches at a higher level try to map multiple biological data levels into one mechanistic network to improve representativeness of data (Chen et al., 2008; Bowick and McAuley, 2011; Amiri et al., 2013; Depiereux et al., 2015; Paraboschi et al., 2015). The generated multi-dimensional network is likely to be more useful in inferring universally involved processes or pathways regardless of inter-studies differences (Azimzadeh Jamalkandi et al., 2015).

Having in mind the common concerns in meta-analysis, we horizontally integrated nine high-throughput transcriptome datasets to identify consensus DEGs. The underlying molecular network in rabies pathogenesis was then extracted based on protein-protein interaction network (PPIN) and signaling pathways by defining the identified DEGs as seed genes. Finally, using real-time PCR, we experimentally validated a number of key DEGs in rabies-infected cells. We demonstrate that a systems biomedicine approach, based on integrating omics datasets and experimental validation, may be used to shed light on a vague portrait of a complex disease pathobiology.

\section{METHODS}

\section{Super Horizontal Integration Datasets}

We looked into all databases pertaining to microarray data at both levels of mRNA and microRNA. This was done by searching databases [Gene Expression Omnibus (GEO), ArrayExpress, Google Scholar, and PubMed NCBI] and studies regarding the rabies virus were extracted with rabies-related keywords including "rabies", "RABV", and "rhabdoviridae" (Figure 1A). Out of a total of 13 studies, 9 were selected for further analysis. The list of included studies and their respective features are given in Supplementary Table 1. In the majority of studies, "brain" and "brain spinal" were the tissues under investigation, and the rest were examined on Mus musculus-derived microglial cells. It should also be noted that only two out of nine inclusive studies were conducted on both mRNA and microRNA levels, with others analyzing only one level of data. Four, three, and two of 

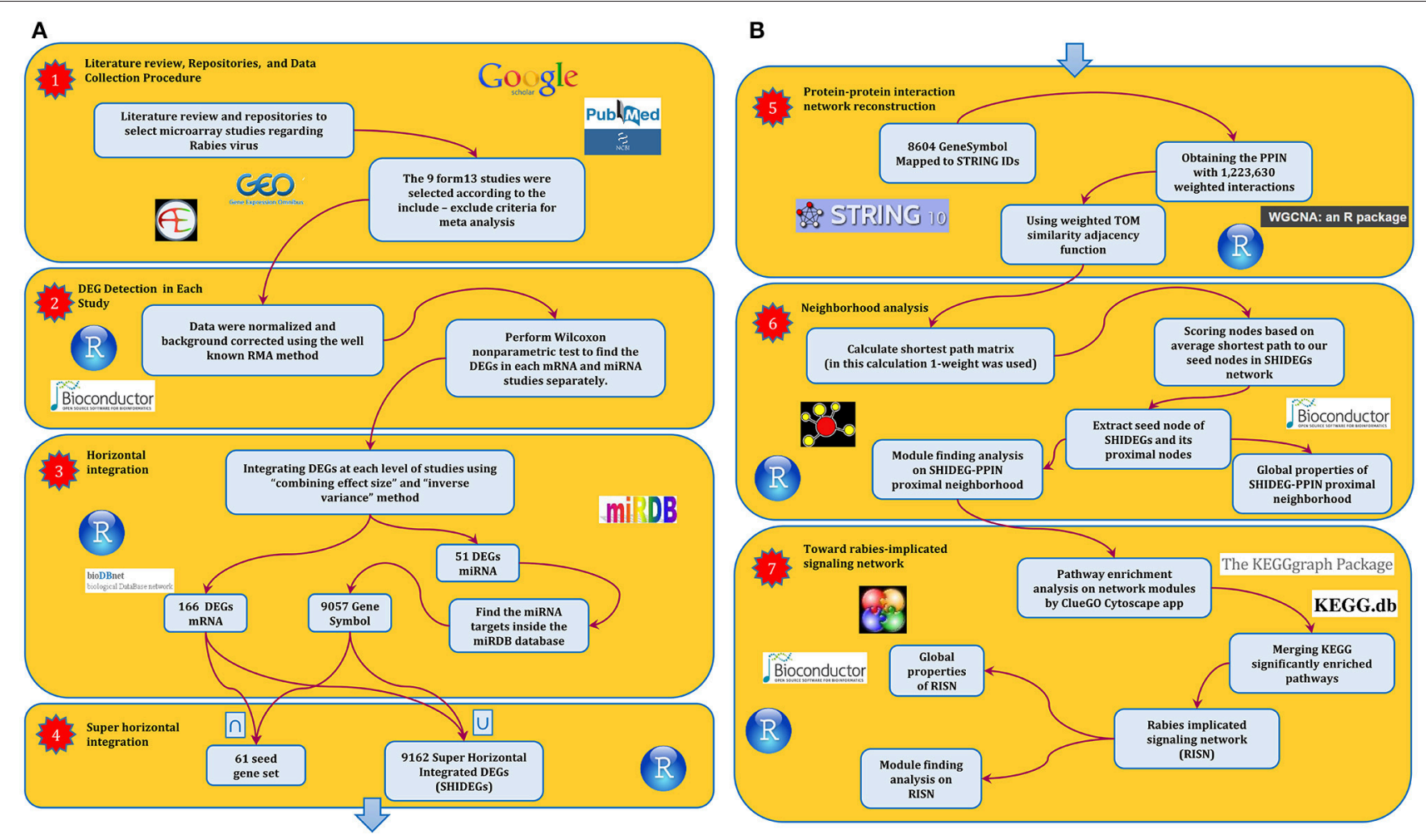

FIGURE 1 | The abstract flowchart of this study design. (A) The four steps taken to obtain the primitive interaction network of the RABV infection. The first step was a systematic review of the literature regarding the RABV. GEO was used to obtain the expression values of studies at the two levels of expression profiling by array of coding and non-coding RNA. Having selected the studies according to our data integration criteria, we moved on to the second step in which we detected DEGs at each level using non-parametric methods. The third step included integration of results using the meta-analysis techniques described in Ramasamy et al. (2008). The implemented integration method revealed that 166 mRNA and 51 microRNA (9057 microRNA targets) are differentially expressed. The next step consisted of super horizontal integration of all transcriptome data. Finally, the 9162 expressed genes were mapped to STRING $v 10.0$ for further analysis. (B) The next stage of the approach which comprised three steps resulted in the rabies-implicated signaling network (RISN). Firstly, the PPIN of all 9162 genes was reconstructed using STRING v10. The combined score calculated in STRING was used as edge weight in the SHIDEG-PPIN. In the second step, the proximal nodes of the seed gene set (nodes) in the whole SHIDEG-PPIN were found to create the seed neighborhood network. Module finding was undertaken along with the global network analysis. Next, the significantly enriched signaling pathways were extracted in the network modules separately. These pathways were then merged together, forming the rabies-implicated signaling network (RISN). Finally, analysis the global network and functional module finding analysis was performed followed by biological inference.

these studies were performed on samples infected by CVS-11, FJDRV and ERA, and RABV-Cre, respectively.

\section{Data Normalization}

In order to prepare data for integration and detect DEGs, it is necessary to use preprocessed and background corrected microarray-data (Ramasamy et al., 2008). First, we checked the quality of recorded CEL format data all of which required to be normalized. The data were normalized by using the "Affy" package in Bioconductor (Gautier et al., 2004). This includes between and within array normalization which reduces the effect of noise and contributes to data consistency. The MA plot and qq-plot for the pairs of samples in each study was analyzed separately to check the normality of data after normalization as a quality control step. Although the qq-plot of some studies revealed that the normalization methods had worked fine, normalization was not successful in datasets with significantly small sample sizes mainly because normality assumptions are violated in low sample size studies.

\section{Analysis of Differentially Expressed Genes (DEGs)}

Assuming that microarray data are normally distributed, the routine procedure to detect DEGs is to perform $t$ test, however, this may result in misleading conclusions if the normality assumption is violated. A recent study showed that oligonucleotide expression values, resulting from widely acceptable calculation methods, are not normally distributed (Hardin and Wilson, 2009). This suggests that the results of $t$-test are biased and unreliable, especially when the sample size in each group is significantly small, and more robust methods should be implemented. Here, we used the Wilcoxon-Mann-Whitney non-parametric test as an alternative method to identify DEGs with the significance level set to 0.05 . The next step was to remove the unmapped probes and solve the problem of "many-to-many conversion" as described in Ramasamy et al. (2008). This was done for both studies of mRNA and microRNA. The computational scripts plus an example of raw data are provided in Data Sheet 1. 


\section{Meta-Analysis}

The results at each level (mRNA and microRNA) were integrated separately, using the inverse-variance technique and combining effect sizes as described in Ramasamy et al. (2008). For integration purposes, the list of DEGs in each study was gathered and the effect size value of each gene was then calculated. We only selected genes with an absolute effect size value $>0.8$ or those with a fold-change $<0.33$ or $>1.5$ (in at least one study) as the frequently accepted cut-off for fold-change. We obtained two different lists of significant differentially expressed values for mRNA and microRNA, respectively. We obtained the target gene symbols of each microRNA accession ID using miRDB (http://mirdb.org; Wong and Wang, 2015). To be best of our knowledge, for Mus musculus, this is the most up-todate repository to convert accession IDs. Finally, after these two parallel horizontal integrations, the union and intersection of the results were extracted as super-horizontally integrated DEGs (SHIDEGs) and the seed gene set of SHIDEGs, respectively.

\section{Background Network Construction}

We used the STRING database to constructed a large-scale PPIN from seven available interaction sources and chose the lowest cut-off for combined scores (Downloaded on 2 September 2015; Szklarczyk et al., 2014). A total of 8604 proteins based on 9162 SHIDEGs were represented in STRING. Accordingly, a total of $1,223,630$ edges were extracted and the STRING combined scores were used as edge weights. Next, the weighted adjacency matrix was transformed to a new adjacency matrix using topological overlapping measure (TOM) function in WGCNA package of R software (Yip and Horvath, 2007; Langfelder and Horvath, 2008; Song et al., 2012). It should be noted that the TOM transformation increases the non-zero adjacency matrix elements as well as very low weight values in this case. The transformed weight distributions of STRING default cut-offs, from lowest to highest confidence, were thus considered to define a new threshold. The third quartile of transformed scores (0.4577) of the highest confidence was selected to strictly filter weak and false-positive interactions.

\section{Neighborhood Ranking}

Using the custom igraph package in $\mathrm{R}$, we generated a matrix of all shortest paths between all pairs of nodes in a weighted network with the algorithm of Dijkstra (Csardi and Nepusz, 2006). First, we substituted raw weights with one-weight to increase reachability of nodes with high weights to seed gene set (nodes) in the shortest path finding procedure. We then defined a distance score, $D_{j}$, for each node in the PPIN as the difference in average of the shortest path to the node when starting on a non-seed node compared with when starting on a seed node, normalized by the average shortest path to reach the node from the whole network.

$$
D_{j}=\frac{\frac{\sum_{i \notin S} S P_{i j}}{|N S|}-\frac{\sum_{i \in S} S P_{i j}}{|S|}}{\frac{\sum_{i} S P_{i j}}{|S|+|N S|}}
$$

Here $S$ is the set of nodes that fall into the seed gene set and NS is the set of nodes that are non-seed nodes. Therefore, a score greater than zero implies that node $j$ falls closer on average to the seed nodes than it does on average to the rest of the network. The rabies network was generated based on the SHIDEGs seed gene set and each member of the seed gene set by scoring all nodes in the network and using a cutoff score of zero to define the neighborhood. It should be noted that the $D$ scores were calculated without imposing any threshold on edge weights.

\section{Undirected PPIN; Topological and Pathway Enrichment Analysis}

To reconstruct a high confidence PPIN around our seed gene set, we used the 0.4577 threshold to filter weak interaction among neighborhood nodes. This filtering resulted in the proximal neighborhood network of seeds. Using Gephi version 0.9, the global topological properties of the resulting PPIN along with module identification was analyzed. To undertake enrichment analysis among the detected modules, ClueGO 2.1.7 (Bindea et al., 2009) in Cytoscape 3.2.1 was used based on Mus musculus using the following parameters: KEGG (Kanehisa et al., 2014), Reactome (Croft et al., 2014), and Wikipathway ontology databases (Kelder et al., 2012), default term selection options, hypergeometric test and Bonferroni step-down $p$-value correction.

\section{Signaling Network Analysis}

The rabies-implicated signaling network (RISN) was constructed based on the KEGG pathways enriched in the rabies PPIN. All statistically significant and frequent pathways in all PPIN modules were extracted and merged together to build a largescale RISN. All SHIDEGs were then delineated in this network by different color labeling. After reviewing clinical and physiological evidences pertaining to the RABV, the whole RISN was delineated into a less complex network.

\section{Cell Culture and Virus}

The Neuro-2a cell line, a murine neuroblastoma cell line, and CVS-11 strain of the RABV (the challenge virus standard) were obtained from the WHO collaborating center for reference and research on rabies, Pasteur Institute of Iran (Tehran, Iran). Virus titers were determined by a focal infectivity assay using BSR (a line of BHK) cells. Neuro-2a cells were grown in Dulbecco's Modified Eagle Medium (DMEM) containing $4500 \mathrm{mg} / \mathrm{L}$ glucose and sodium bicarbonate, supplemented with $10 \%$ fetal bovine serum. Cultures were maintained at $37 \mathrm{C}$ in a $5 \% \mathrm{CO}_{2}$ humidified cell incubator with growth medium replaced every $48 \mathrm{~h}$. For all experiments, cells were subcultured into $25 \mathrm{~cm}$ tissue culture flasks and were grown for $16 \mathrm{~h}$ before infection.

\section{Total RNA Isolation, cDNA Synthesis, and Primer Design for PCR}

Total mRNA was isolated from neuroblastoma cells (mock infected and infected with the CVS-11 strain of RABV) using the RNX RNA Isolation Kit (CinnaGen Inc., Tehran, Iran). The amount and purity of RNA were determined by Biotek microplate spectrophotometry. The extracted RNA was then treated with DNase to remove genomic DNA. 
Total RNA $(1.7 \mu \mathrm{g} / \mathrm{ml})$ was reverse transcribed into firststrand cDNA by the SuperScript III First-Strand Synthesis System (Thermo Fisher Scientific) and oligo(dT)18 according to the manufacturer's protocol. Primer specificity was tested by primer-BLAST (http://blast.ncbi.nlm.nih.gov/Blast.cgi) and experimentally by the positive control amplification. Optimal PCR conditions were identified for each primer pair. GAPDH was used as an internal control for RT-polymerase chain reactions.

\section{Quantitative Real-Time PCR}

Reverse transcription-quantitative real-time PCR (RT-qPCR) was carried out on a Rotor-Gene Q 5plex HRM instrument (Qiagen, Hilden, Germany) with EvaGreen fluorescence dye (Biotium, Hayward, USA) to monitor cDNA amplification of GNAI2, AKT3, IL21, and GAPDH through increased fluorescence intensity. The specificity of the amplified products was checked by melting curve analysis, and the expected size of the fragments was further visualized by gel electrophoresis (2\% agarose) and staining with GelRed (Biotium, Hayward, CA). Results were confirmed by triplicate testing. Relative mRNA expression was calculated using the delta-delta $C t$ method (Livak and Schmittgen, 2001). Sequences were analyzed using Seqscanner. Statistical analysis was performed by depicting an error bar for each gene in each condition to compare relative expression of the abovementioned genes in uninfected and RABV-infected states.

\section{RESULTS}

This study comprises seven steps in two separate parts as illustrated in the Figure 1. After a systematic literature review, nine transcriptomic datasets pertaining to rabies were collected (Supplementary Table 1). DEGs were identified in the mRNA and microRNA datasets and used to construct a PPIN of rabies infection. In the second part, analysis of the PPIN neighborhood and rabies-implicated signaling was implemented to create a mechanistic description of the molecular pathogenesis of rabies infection (Figure 1B). The outcome of each step is discussed in more detail below.

\section{Intersection of mRNA and microRNA Transcriptome Data by Super Horizontal Integration Reveals an Intriguing List of the Seed Gene Set}

A total of 166 DEGs were identified at the mRNA level. Analysis at the microRNA level led to the identification of 51 genes which target 9057 genes on mouse genome. The genes at both mRNA and microRNA levels were combined to create a list of 9162 "super horizontally integrated-DEGs (SHIDEGs)" and a list of 61 intersecting genes considered as "seed genes" (Table 1). Of the 9162 SHIDEGs, 8604 ( 93\%) were mapped to STRING version 10 and the obtained network contained 1,223,630 weighted protein-protein interactions.

Next, we obtained gene ontology (GO) classifications for all seed genes. Using the Enrichr web based tools (Chen et al., 2013; Kuleshov et al., 2016) significantly enriched biological process (BP), molecular function (MF), and cellular component (CC) terms were retrieved and then ranked by combined scores (Figure 2). From a biological process point of view, diverse inflammatory responses such as cytokine-mediated signaling pathway (GO:0019221) and regulation of leukocyte activation (GO:0002694) were enriched. Also, the other moiety of BPs was generally associated with nucleotide biosynthetic processes (Figure 2A). This observation confirmed the role of immune signaling pathways and propagation apparatus in rabies infection. CC and MF enriched terms further accentuated the role of signaling alteration in development of rabies (Figures 2B,C).

\section{Shortest Path-Based Scoring Allows Identification of the Seed Gene Neighborhood in the Protein-Protein Interaction Space}

We applied network concepts to explore more thoroughly the potential functional relationship between the identified DEGs and RABV pathogenesis. We postulated that all integrated DEGs are involved in the global interactome perturbed by the RABV. We assumed that the SHIDEGs are more likely to interact directly with the RABV and the neighbors of SHIDEGs are of the next level of etiological importance. To identify the disease subnetwork of SHIDEGs in PPIN, we retrieved the entire protein-protein weighted interactions from STRING (Szklarczyk et al., 2014). The giant component comprising 8604 DEG products was selected for further analysis. Given the high false-positive rate in PPINs (Jafari et al., 2013, 2015), the topological overlap matrix (TOM)-based adjacency function was used to filter the effect of spurious or weak connections ( $\mathrm{Li}$ and Horvath, 2007; Yip and Horvath, 2007). Proteins encoded by the 61 seed genes were identified in the refined global PPIN for neighborhood analysis.

Based on biological parsimony and the observed patterns in different signaling databases, biological responses are controlled via a short signaling cascade (Gitter et al., 2011; Silverbush and Sharan, 2014). We therefore used the shortest path algorithm to identify nodes in proximity of the seed nodes. We then ranked the nodes within the whole PPIN using distance D. From the total 8602 nodes within the robust PPIN, 3775 nodes had a positive score and therefore fell within the seed gene neighborhood.

Subsequently, to filter the edges having low weight, STRING combined score (weight of edges) were transformed using the TOM-based adjacency function and those above the $3^{\text {rd }}$ quartile were retained. Of all nodes with $D>0,694$ nodes passed this filter and were considered as the "seed gene proximal neighborhood network". This resulted in the selection of highly important relationships among nodes based on network topology (Figure 3).

\section{The Identification of Rabies-Implicated Gene Products}

The final rabies infection PPIN contained 694 nodes with 6097 interactions. The degree distribution (Supplementary Figure 1) and modularity index $(\sim 0.7)$ of this PPIN indicate that it has a modular structure and a scale free topology. Its average path 
TABLE 1 | Seed gene set.

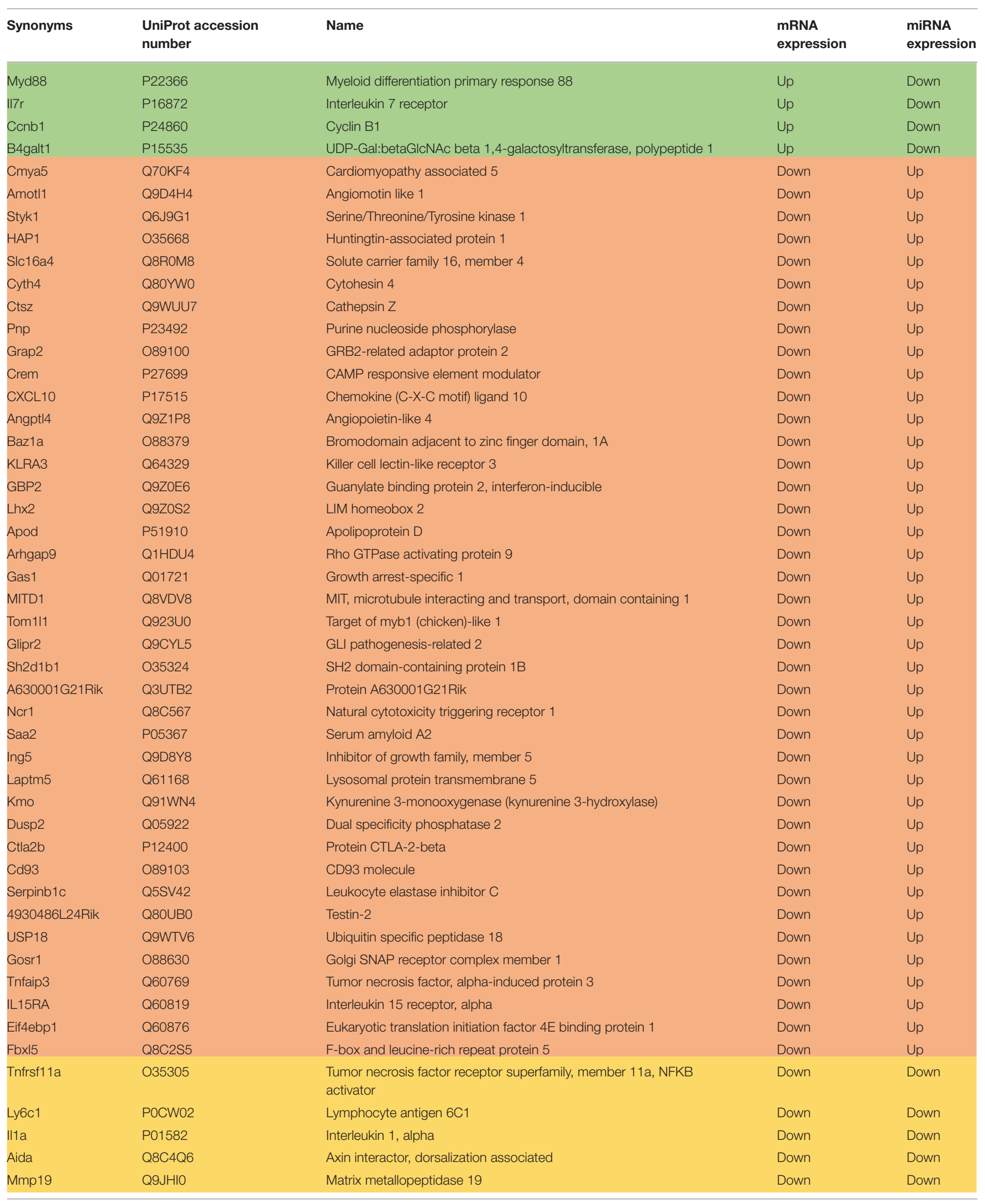


TABLE 1 | Continued

\begin{tabular}{|c|c|c|c|c|}
\hline Synonyms & $\begin{array}{l}\text { UniProt accession } \\
\text { number }\end{array}$ & Name & $\begin{array}{l}\text { mRNA } \\
\text { expression }\end{array}$ & $\begin{array}{l}\text { miRNA } \\
\text { expression }\end{array}$ \\
\hline Csf1 & P07141 & Colony stimulating factor 1 (macrophage) & Down & Down \\
\hline Arrdc4 & Q0GJK1 & Arrestin domain containing 4 & Down & Down \\
\hline Nampt & Q99KQ4 & Nicotinamide phosphoribosyltransferase & Down & Down \\
\hline Nfkb2 & Q9WTK5 & $\begin{array}{l}\text { Nuclear factor of kappa light polypeptide gene enhancer in B-cells } 2 \\
\text { (p49/p100) }\end{array}$ & Down & Down \\
\hline Fkbp5 & Q64378 & FK506 binding protein 5 & Down & Down \\
\hline KIhl5 & Q6PFE1 & Kelch-like family member 5 & Up & Up \\
\hline Snx10 & Q9CWT3 & Sorting nexin 10 & Up & Up \\
\hline SERPINB9 & 008797 & Serpin peptidase inhibitor, clade B (ovalbumin), member 9 & Up & Up \\
\hline IGF2 & P09535 & Insulin-like growth factor 2 & Up & Up \\
\hline IFI204 & P15092 & Interferon-activable protein 204 & Up & Up \\
\hline "l17ra & Q60943 & Interleukin 17 receptor $A$ & Up & Up \\
\hline Zc3h12a & Q5D1E7 & Zinc finger $\mathrm{CCCH}$-type containing $12 \mathrm{~A}$ & Up & Up \\
\hline
\end{tabular}

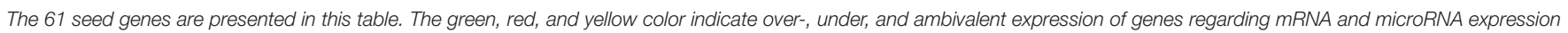
evidence.

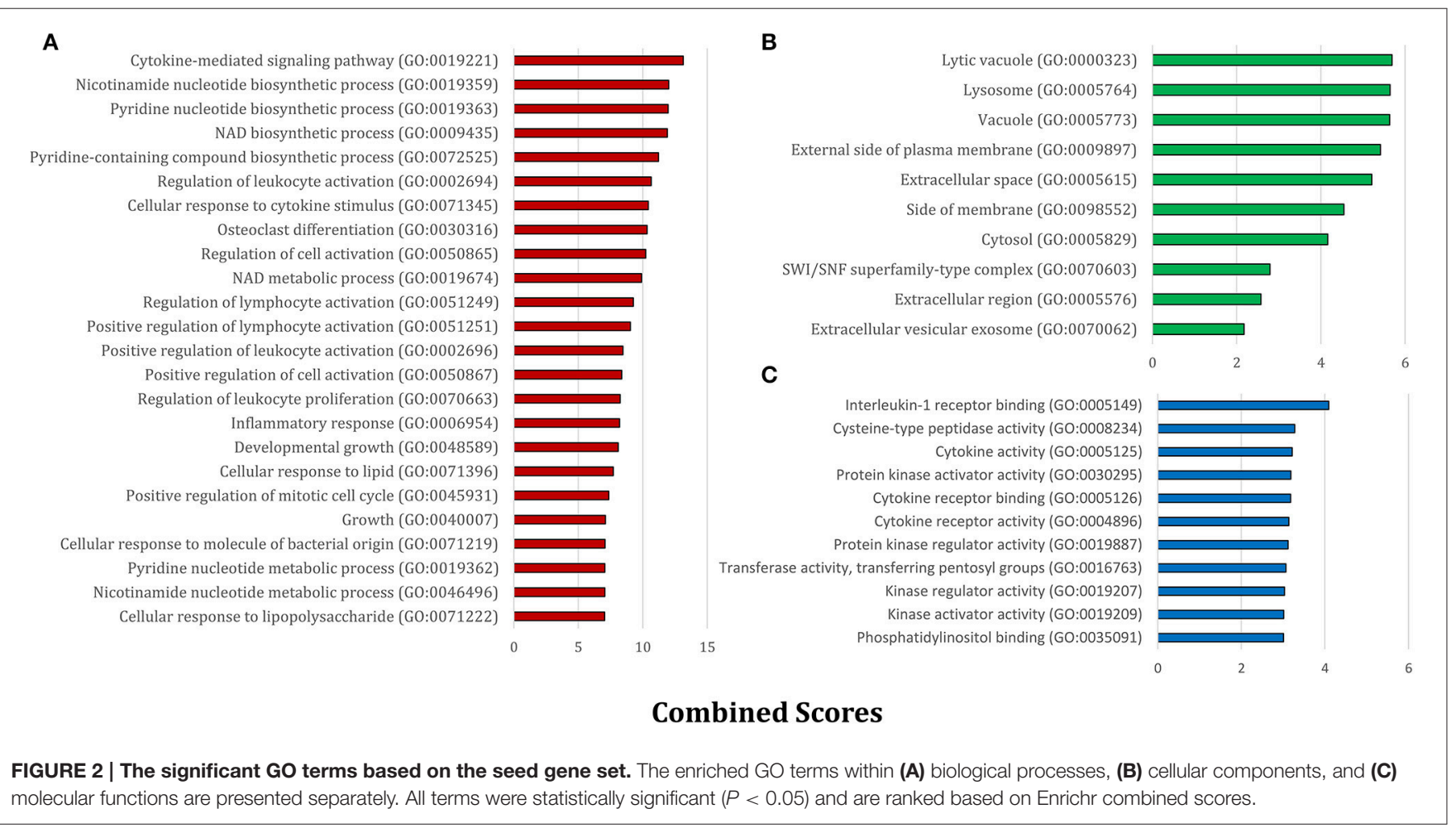

length and diameter were 5.16 and 14 , respectively, showing that this relatively large and sparse network is small-world. To infer the functionality of this refined network, we analyzed the network modules. Twelve modules were detected by the fast unfolding clustering algorithm implemented in Gephi (V. 0.9) (Bastian et al.).

To avoid bias in inferring global properties of the network, the top three central nodes in each module were specifically shown in Figure 4. This Figure illustrates these nodes in terms of degree and betweenness centrality measures. Interestingly, all of these nodes have diverse receptor binding and kinase activity functions based on GO enrichment analysis. On the other hand, our results revealed that inter-modular high-degree nodes related to CCR chemokine receptor binding (GO:0048020), R-SMAD binding (GO:0070412), responses to mechanical stimulus (GO:0009612), JAK-STAT cascade involved in growth hormone signaling pathway (GO:0060397), and negative regulation of neuron death (GO:1901215) were down-regulated by the RABV. In contrast, the local and global hub proteins associated with positive regulation of protein kinase activity (GO:0045860), 

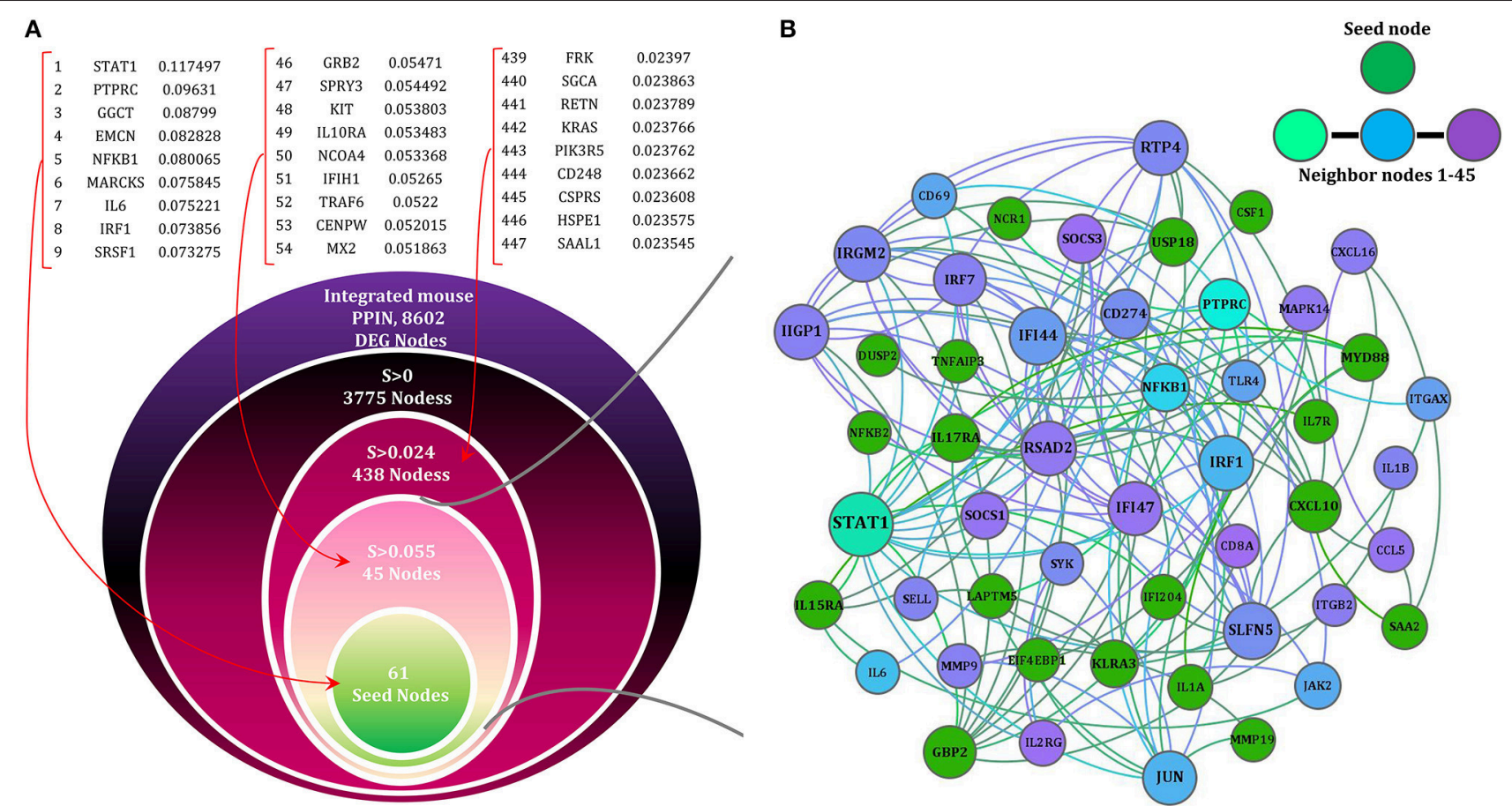

FIGURE 3 | The network (SHIDEG-PPIN) onion diagram. (A) Identification of the rabies disease neighborhood network based on proximal nodes of the seed gene set. The PPIN of all DEG products (8602/9162) existed in STRING were ranked on the basis of their shortest path (SP) score with the 61 rabies SHIDEGs as seed nodes. Selecting various score cutoffs $\left(99.5^{\text {th }}, 95^{\text {th }}\right.$ and $56^{\text {th }}$ quantiles) allow neighborhoods of various sizes to be defined as shown in the nested circles. The ranked list of neighbors of seed nodes demonstrated with their scores. (B) The minimized PPIN formed with the confident interactions (0.4 cut-off selected based on the TOM procedure) between the 61 seed nodes (green) and top 45 ranked proximal nodes (range of colors from cyan to purple) is shown at the top with node size representing degree of nodes.

cellular response to lipid (GO:0071396), neurotrophin TRK receptor signaling pathway (GO:0048011), G-protein coupled receptor binding (GO:0001664), and neuropeptide hormone activity (GO:0005184) were up-regulated, thus facilitating virus survival and propagation by avoiding programmed cell death.

The same scenario also applied to nodes with high betweenness centrality. For example, nodes associated with neurotrophin receptor binding (GO:0005165) and cellular response to organonitrogen compound (GO:0071417) were overexpressed while those associated with natural immune system were underexpressed (Supplementary Table 2). On top of that, the top five ranked nodes based on betweenness centrality, namely EP300, STAT1, RHOA, and PDGFA were underexpressed concurrently. This may lead to a lack of network coordination among different immune processes.

Given the abundance of receptors and kinases in this network, we performed pathway enrichment analysis on each module separately. Using ClueGO (Cytoscape plugin; Bindea et al., 2009), the statistically significant pathway terms were identified among those in the Kyoto Encyclopedia of Genes and Genomes (KEGG; Kanehisa et al., 2014) and Reactome (Croft et al., 2014) databases (Table 2 and Supplementary Table 3). We then ranked the enriched pathway terms based on gene coverage (Ansari-Pour et al., 2016).
Furthermore, to evaluate the quality of module discovery results, conformity of enriched pathways in a module was assessed with respect to the interconnectedness level of that module (Supplementary Table 4). Our results demonstrated that the KEGG enriched pathway similarity matrix was significantly correlated with the module interconnectivity matrix $(P<0.01)$ and that they were highly similar (Rand measure $=73 \%$ ).

\section{Toward Identifying the Signaling Network Involved in Rabies Pathogenesis}

In order to retrieve casual relationships, we used the KEGG database and enrichment results to prune the proximal network of the seed gene set. By reviewing the significantly enriched pathways, all KEGG pathways $(N=47$; Supplementary Table 3$)$ were merged to reconstruct the enriched signaling network pertaining to rabies pathogenesis. The full signaling network is presented in Supplementary Table 5, but the merge of only 22 of them were presented in Figure 5. These 22 pathways were selected based on gene coverage, reported relevance to rabies pathogenesis and association with other viral infections. Then, the DEGs related to these pathways were used to mine the rabies-implicated signaling network (RISN) based on the following KEGG pathways: PI3K-AKT signaling pathway (KEGG:04151), cell cycle (KEGG:04110), JakSTAT signaling pathway (KEGG:04630), circadian rhythm 


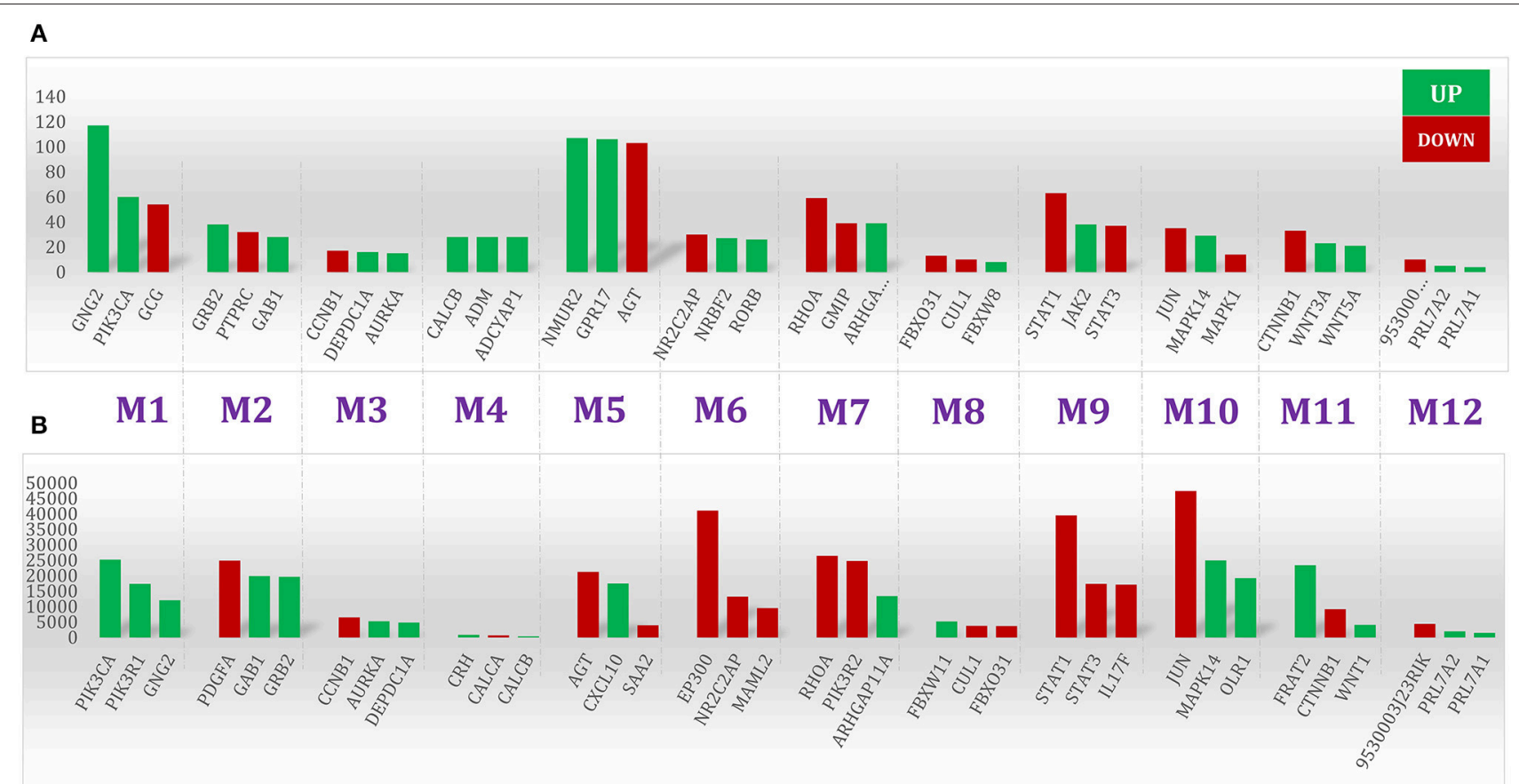

FIGURE 4 | Top ranked nodes based on two centrality measures. The (A) degree and (B) betweenness centrality measures were calculated in the 12 modules (M1-M12) of the SHIDEG-PPIN proximal neighborhood network of the seed gene set. The over-expression and under-expression of these gene products are labeled by color.

(KEGG:04710), pertussis (KEGG:05133), leishmaniasis (KEGG:05140), tuberculosis (KEGG:05152), hepatitis B (KEGG:05161), influenza A (KEGG:05164), herpes simplex infection (KEGG:05168), Epstein-Barr virus infection (KEGG:05169), inflammatory bowel disease (IBD) (KEGG:05321), PPAR signaling pathway (KEGG:03320), hematopoietic cell lineage (KEGG:04640), neuroactive ligandreceptor interaction (KEGG:04080), Notch signaling pathway (KEGG:04330), inflammatory mediator regulation of TRP channels (KEGG:04750), TNF signaling pathway (KEGG:04668), $\mathrm{T}$ cell receptor signaling pathway (KEGG:04660), cytokinecytokine receptor interaction (KEGG:04060), chemokine signaling pathway (KEGG:04062), and ubiquitin mediated proteolysis (KEGG:04120). The main sink and source nodes in this directed network along with the nodes with high betweenness centrality in the whole RISN are listed in Table 3. The influence of nodes with high betweenness on propagating or focusing information among this signaling network is presented by the information release index (IRI), $I R I=\log ($ Outdegree/Indegree $)$. The positive value of IRI indicates the propagating role of nodes and vice versa.

\section{Manually Curated Version of RISN}

To simplify RISN, signaling pathways were manually extracted and merged based on the currently available data in KEGG, including WNT, MAPK/ERK, RAS, PI3K/AKT, Toll-like receptor, JAK/STAT, and NOTCH signaling pathways. The information flow from diverse ligands to various transcription factors is illustrated along with differential expression. As shown in Figure 6, information is converged toward several important proteins including PLC, MAPK1/2, PIK3, PKC, and JAK, and is then diverged toward several distinct transcription factors and finally end-point biological processes.

Our analysis revealed that two of three WNT signaling pathways were altered in rabies infected cells. The canonical WNT pathway (WNT/ $\beta$-catenin) along with the non-canonical planar cell polarity (PCP) pathway were apparently active in infected neurons but the non-canonical WNT/calcium pathway was not induced. The PCP pathway is involved in up-regulation of components of the downstream pathway and cytoskeletal rearrangements of which the latter may implicate this pathway in cytoskeletal changes in neurons. This is consistent with previous studies reporting cooperative cytoskeletal changes (restructuration) for viral protein transportation and viral localization (Sagara et al., 1995; Ceccaldi et al., 1997; Song et al., 2013; Zandi et al., 2013).

There is also evidence of crosstalk between WNT and MAPK/ERK signaling pathways. It seems that in the rabid brains the MAPK/ERK signaling pathway, via CAMP-PCREB signaling, is involved in neuromelanin biosynthesis of which its accumulation depletes iron ions as observed in some neurodegenerative diseases such as Parkinson's disease (Good et al., 1992). Iron deficiency may also contribute to defective dopaminergic interaction with neurotransmission systems (Youdim, 2008). This is, however, a speculation and needs experimental validation in rabies infection cases.

Additionally, RAS signaling is activated through the C-Kit receptor and diverge toward PIK3 and MAPK/ERK signaling 
TABLE 2 | The enriched KEGG and Reactome pathways.

\begin{tabular}{|c|c|c|c|c|c|c|}
\hline $\begin{array}{l}\text { Module } \\
\text { No. }\end{array}$ & KEGG & Frequency & $\begin{array}{c}P \text {-value corrected } \\
\text { with Bonferroni step } \\
\text { down }\end{array}$ & Reactome & Frequency & $\begin{array}{c}P \text {-value corrected } \\
\text { with Bonferroni step } \\
\text { down }\end{array}$ \\
\hline & Calcium signaling pathway & 21 & $2.76 \mathrm{E}-28$ & Platelet activation, signaling, and aggregation & 10 & 2.76E-08 \\
\hline & cGMP-PKG signaling pathway & 7 & $2.56 \mathrm{E}-05$ & $\begin{array}{l}\text { Thrombin signaling through proteinase } \\
\text { activated receptors (PARs) }\end{array}$ & 7 & $2.49 \mathrm{E}-10$ \\
\hline \multirow[t]{3}{*}{ M2 } & PI3K-Akt signaling pathway & 30 & $2.16 \mathrm{E}-24$ & Immune system & 49 & $9.78 \mathrm{E}-28$ \\
\hline & Pathways in cancer & 30 & 1.26E-22 & Innate immune system & 38 & $1.31 E-25$ \\
\hline & Ras signaling pathway & 29 & $2.10 \mathrm{E}-28$ & Adaptive immune system & 35 & $6.87 \mathrm{E}-23$ \\
\hline M3 & Cell cycle & 10 & $1.71 \mathrm{E}-11$ & Cell cycle & 28 & $4.52 E-31$ \\
\hline \multirow[t]{3}{*}{ M4 } & $\begin{array}{l}\text { Neuroactive ligand-receptor } \\
\text { interaction }\end{array}$ & 16 & $3.08 \mathrm{E}-22$ & G alpha (s) signaling events & 23 & $1.04 \mathrm{E}-47$ \\
\hline & - & - & - & GPCR ligand binding & 21 & $1.91 \mathrm{E}-29$ \\
\hline & - & - & - & Class B/2 (secretin family receptors) & 10 & $5.73 \mathrm{E}-16$ \\
\hline \multirow[t]{3}{*}{ M5 } & Chemokine signaling pathway & 25 & $1.78 \mathrm{E}-29$ & Signaling by GPCR & 68 & $2.40 \mathrm{E}-78$ \\
\hline & $\begin{array}{l}\text { Neuroactive ligand-receptor } \\
\text { interaction }\end{array}$ & 25 & $2.85 E-25$ & GPCR downstream signaling & 63 & $2.90 \mathrm{E}-70$ \\
\hline & $\begin{array}{l}\text { Cytokine-cytokine receptor } \\
\text { interaction }\end{array}$ & 20 & 4.75E-18 & G alpha (i) signaling events & 63 & $1.24 \mathrm{E}-110$ \\
\hline M6 & $\begin{array}{l}\text { Thyroid hormone signaling } \\
\text { pathway }\end{array}$ & 12 & $1.56 \mathrm{E}-12$ & Generic transcription pathway & 46 & $3.10 \mathrm{E}-54$ \\
\hline M7 & - & - & - & G alpha (12/13) signaling events & 17 & $1.45 \mathrm{E}-25$ \\
\hline \multirow[t]{3}{*}{ M8 } & Ubiquitin mediated proteolysis & 8 & $2.36 \mathrm{E}-12$ & $\begin{array}{l}\text { Association of TriC/CCT with target proteins } \\
\text { during biosynthesis }\end{array}$ & 4 & $1.40 \mathrm{E}-07$ \\
\hline & Circadian rhythm & 2 & 1.19E-03 & Protein folding & 4 & $1.44 \mathrm{E}-06$ \\
\hline & - & - & - & Chaperonin-mediated protein folding & 4 & $1.04 \mathrm{E}-06$ \\
\hline \multirow[t]{3}{*}{ M9 } & Jak-STAT sianalina pathwav & 37 & $2.06 \mathrm{E}-50$ & Immune system & 57 & $3.06 \mathrm{E}-41$ \\
\hline & $\begin{array}{l}\text { Cytokine-cytokine receptor } \\
\text { interaction }\end{array}$ & 36 & 1.80E-39 & Cytokine signaling in immune system & 53 & $1.54 \mathrm{E}-67$ \\
\hline & Measles & 26 & $9.44 \mathrm{E}-32$ & Interferon signaling & 31 & 1.08E-35 \\
\hline \multirow[t]{3}{*}{ M10 } & MAPK sianalina pathwav & 25 & $3.59 \mathrm{E}-24$ & Innate immune system & 31 & $2.54 \mathrm{E}-21$ \\
\hline & Pathways in cancer & 23 & $7.36 \mathrm{E}-17$ & Toll-like receptors cascades & 19 & $1.54 \mathrm{E}-20$ \\
\hline & PI3K-Akt signaling pathway & 22 & $7.01 \mathrm{E}-17$ & Toll like receptor 3 (TLR3) cascade & 18 & 7.97E-22 \\
\hline \multirow[t]{3}{*}{ M11 } & Wht sianalina pathwav & 33 & $6.80 \mathrm{E}-57$ & Signaling by Wnt & 30 & $1.06 \mathrm{E}-37$ \\
\hline & Pathways in cancer & 28 & 2.05E-31 & TCF dependent sianalina in response to WNT & 23 & $1.72 \mathrm{E}-28$ \\
\hline & Melanogenesis & 26 & $2.21 \mathrm{E}-44$ & Class B/2 (secretin family receptors) & 18 & 1.63E-27 \\
\hline \multirow[t]{2}{*}{ M12 } & - & - & - & Amyloids & 5 & $1.21 \mathrm{E}-09$ \\
\hline & - & - & - & Disease & 5 & 8.05E-05 \\
\hline
\end{tabular}

The statistically significantly enriched KEGG and Reactome pathways were identified by ClueGO. The top three representative pathways identified in each module (M1-M12) of the SHIDEG-PPIN proximal neighborhood network are given together with their corrected p-values. The highlighted pathway names were found to be enriched in more than one module. 

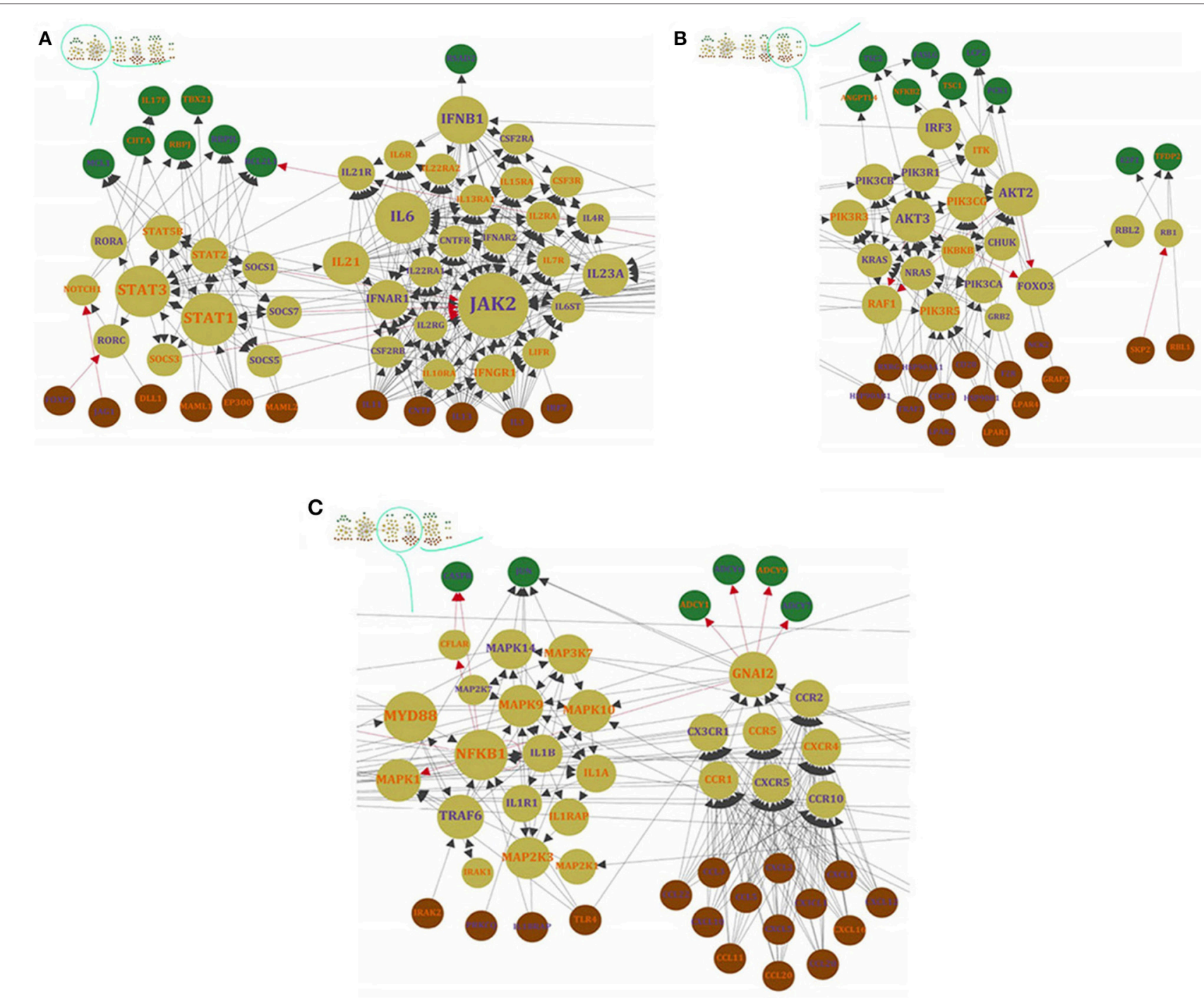

FIGURE 5 | A bird's eye view of RISN. The 22 over-expressed genes of significantly enriched KEGG pathways were selected and merged. The DEGs are labeled by different colors including orange and blue which indicates down-regulation and up-regulation of genes, respectively. The activator/inhibitor edges are also colored differently (red edges are inhibitors and black vice versa). The node colors represent its relative position in a directed network from brown (source nodes), cream (internal nodes) to green (sink nodes) and the node size is proportional to betweenness centrality value. In addition, the six detected modules in three parts are displayed separately: (A) Interferon circumvent, (B) Toward proliferation and survival, and (C) neuropathological clue.

pathways. Downstream of RAS activation (ERK signaling and AKT) is highly complex but generally contributes to cell growth (Bender et al., 2015). Activated RAS signaling suppresses PKR-mediated responses to interferon response and double-stranded RNA degradation. Normally, viral transcripts trigger PKR phosphorylation and activation, and finally inhibit infection. Therefore, the RABV may replicate silently in RAS activated cells (Mundschau and Faller, 1994; Russell, 2002). This data-based hypothesis also requires experimental validation in rabid cases.

The AKT signaling pathway plays a critical role in the replication of the RABV similar to other non-segmented negative-stranded RNA viruses. Heavy phosphorylation of viral proteins (P protein) is mainly mediated via AKT activity (Sun et al., 2008). Subsequently, the activated P protein plays a crucial role in other signaling pathways such as Tolllike receptor and JAK/STAT signaling pathways which are responsible for viral genome detection and immune-modulatory functions against rabies, respectively. Accordingly, the viral G protein activates AKT signaling through phosphorylation and localization of PTEN (Terrien et al., 2012). The consequences of the activation and crosstalk of these signaling pathways are reduced apoptosis, cell survival and blocked cell cycle progression. Neuronal dysfunction, inhibition of apoptosis, and limitation of inflammation have been previously stressed by Gomme et al. (2012). It seems that these processes have been evolutionarily acquired to complete virus lifecycle and transfer to the new host. They also showed that most of DEGs are involved in signaling transduction and nervous system function, and therefore affect cell behavior by decreasing neurite growth, 
TABLE 3 | Details of the main sink and source nodes along with high betweenness centrality values in the whole RISN.

\begin{tabular}{llll}
\hline Gene symbol & Protein name & Po. & U/D \\
\hline CCL11 & Chemokine (C-C motif) ligand 11 & Source & Down \\
CCL20 & Chemokine (C-C motif) ligand 20 & Source & Down \\
CCL22 & Chemokine (C-C motif) ligand 22 & Source & Up \\
CCL28 & Chemokine (C-C motif) ligand 28 & Source & Up \\
CCL3 & Chemokine (C-C motif) ligand 3 & Source & Up \\
CCL5 & Chemokine (C-C motif) ligand 5 & Source & Up
\end{tabular}

Role in rabies

$\begin{array}{llll}\text { CD28 } & \text { CD28 molecule } & \text { Source } & \text { Up } \\ \text { CDC37 } & \text { Cell division cycle 37 } & \text { Source } & \text { Up } \\ \text { CNTF } & \text { Ciliary neurotrophic factor } & \text { Source } & \text { Up } \\ \text { CX3CL1 } & \text { Chemokine (C-X3-C motif) ligand 1 } & \text { Source } & \text { Up } \\ \text { CXCL1 } & \text { Chemokine (C-X-C motif) ligand 1 (melanoma growth } & \text { Source } & \text { Up } \\ & \text { stimulating activity, alpha) } & & \\ \text { CXCL10 } & \text { Chemokine (C-X-C motif) ligand 10 } & \text { Source } & \text { Up }\end{array}$

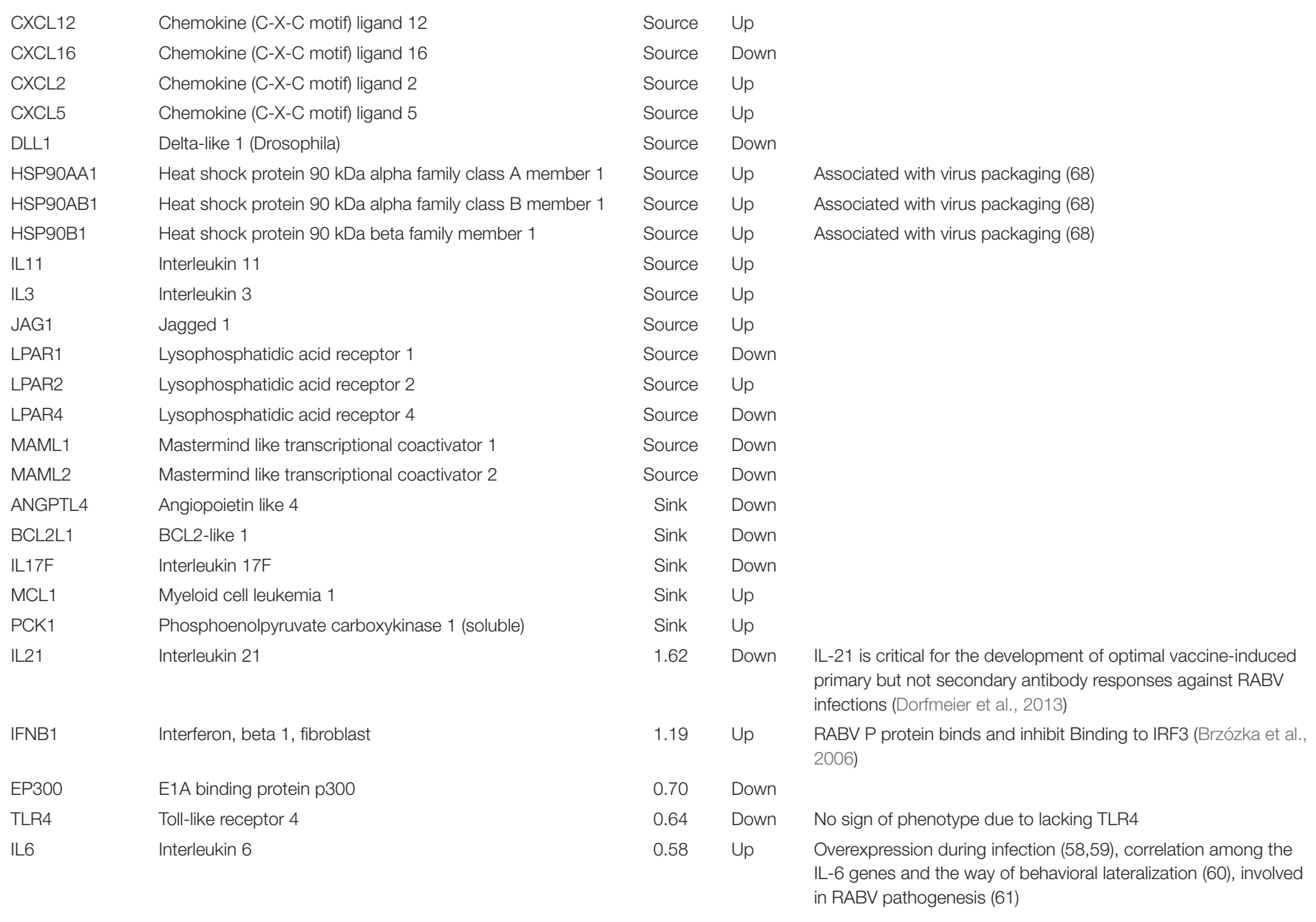

Known as a vital regulator which is involved in convincing encephalomyelitis (72), raised levels of mRNA transcripts (73), the expression value of CXCL10 and CCL5 in microglia is accurately regulated while the multiple signaling pathways are activated

Known encephalomyelitis (72), raised levels of mRNA transcripts (73), the expression value of CXCL10 and CCL5 in microglia is accurately regulated while the multiple signaling pathways are activated

Associated with virus packaging (68)

Associated with virus packaging (68)

Heat shock protein $90 \mathrm{kDa}$ alpha family class B member

Source Up

Source Up

Source Up

Source Down

Source Up

Source Down

Source Down

Source Down

Sink Down

Sink Down

Sink Down

Sink Up

Sink Up RABV P protein binds and inhibit Binding to IRF3 (Brzózka et al., No sign of phenotype due to lacking TLR4

(Continued) 
TABLE 3 | Continued

\begin{tabular}{|c|c|c|c|c|}
\hline Gene symbol & Protein name & Po. & U/D & Role in rabies \\
\hline MYD88 & Myeloid differentiation primary response 88 & 0.44 & Down & $\begin{array}{l}\text { Weakened RABV intervenes deadly disease while no MyD } 88 \\
\text { present, genetic adjuvanting with Myd88 improved the RVNA } \\
\text { responses of a plasmid DNA rabies vaccine }(90,91)\end{array}$ \\
\hline NFKB1 & $\begin{array}{l}\text { Nuclear factor of kappa light polypeptide gene enhancer in } \\
\text { B-cells } 1\end{array}$ & 0.13 & Down & \\
\hline STAT3 & $\begin{array}{l}\text { Signal transducer and activator of transcription } 3 \\
\text { (acute-phase response factor) }\end{array}$ & 0.12 & Down & inhibits STAT3 nuclear accumulation (Lieu et al., 2013) \\
\hline F2R & Coagulation factor II (thrombin) receptor & 0.05 & Up & \\
\hline CASP8 & Caspase 8, apoptosis-related cysteine peptidase & 0.00 & Up & Activation in RABV (Sarmento et al., 2006) \\
\hline GNAI2 & $\begin{array}{l}\text { Guanine nucleotide binding protein ( } G \text { protein), alpha } \\
\text { inhibiting activity polypeptide } 2\end{array}$ & 0.00 & Down & \\
\hline RBL1 & Retinoblastoma-like 1 & 0.00 & Down & \\
\hline AKT2 & v-AKT murine thymoma viral oncogene homolog 2 & -0.07 & Up & Hyper-phosphorylation of RABV P protein (Sun et al., 2008) \\
\hline AKT3 & v-AKT murine thymoma viral oncogene homolog 3 & -0.07 & Up & Hyper-phosphorylation of RABV P protein (Sun et al., 2008) \\
\hline JUN & Jun proto-oncogene & -0.32 & Up & Activated in Rabies (Nakamichi et al., 2005) \\
\hline FASLG & Fas ligand (TNF superfamily, member 6) & -0.48 & Up & $\begin{array}{l}\text { Immune disruptive strategy of RABV to bring about apoptosis in } \\
\text { T cell by overexpression in neuron (93) }\end{array}$ \\
\hline IRF7 & Interferon regulatory factor 7 & -0.60 & Up & RABV P protein averts tis activation (70) \\
\hline ADCY6 & Adenylate cyclase 6 & -0.70 & Up & $\begin{array}{l}\text { The signal pathway from the stimulating regulatory component } \\
\text { of the adenylate cyclase system to the unchanged activity of the } \\
\text { catalytic subunit is defective (Koschel and Halbach, 1979; } \\
\text { Koschel and Münzel, 1984) }\end{array}$ \\
\hline ADCY1 & Adenylate cyclase 1 (brain) & -0.73 & Down & $\begin{array}{l}\text { The signal pathway from the stimulating regulatory component } \\
\text { of the adenylate cyclase system to the unchanged activity of the } \\
\text { catalytic subunit is defective (Koschel and Halbach, 1979; } \\
\text { Koschel and Münzel, 1984) }\end{array}$ \\
\hline IRF3 & Interferon regulatory factor 3 & -0.78 & Up & $\begin{array}{l}\text { RABV P protein binds and avert binding to IFNB1 (53), inhibit } \\
\text { IRF3 phosphorylation }(70,96)\end{array}$ \\
\hline JAK2 & Janus kinase 2 & -0.79 & Up & \\
\hline
\end{tabular}

The third and fourth column indicate the position (Po.) of the corresponding genes in RISN and expression changes (U/D) of them based on our meta-analysis. The IRI of the nodes with high betweenness values are presented in the third column

organization of cytoskeleton and cytoplasm, and microtubule dynamics.

It has been demonstrated that rabies infection up-regulates expression of CXCL10 and CCL5 proteins in a ERK1/2-, p38-, and NFkB-dependent manner (Nakamichi et al., 2004, 2005). CXCL10 is a major chemo-attractant of Th-1 cells. The up-regulation of interferon, chemokines, interleukin (IL), and IL-related genes were previously observed by Sugiura et al. (2011). They also reported the signaling pathways involved in rabies infection including interferon signaling, IL-15 production and signaling, and Granzyme
B signaling which trigger apoptosis in immune target cells.

ERK and p38MAPK along with BCL2 family and the FasL receptor are important apoptosis committers. Currently, it is known that RISN inhibits apoptosis and also suppresses cell proliferation (Gomme et al., 2012). Also, JAK/STAT signaling is activated through its receptors, but as mentioned earlier STAT dimerization is inhibited via viral $\mathrm{P}$ protein activity. Therefore, downstream signaling of STATs, which is critical for interferon signaling and viral defense, is suppressed. Concomitant activation of JAK/STAT and AKT signaling 


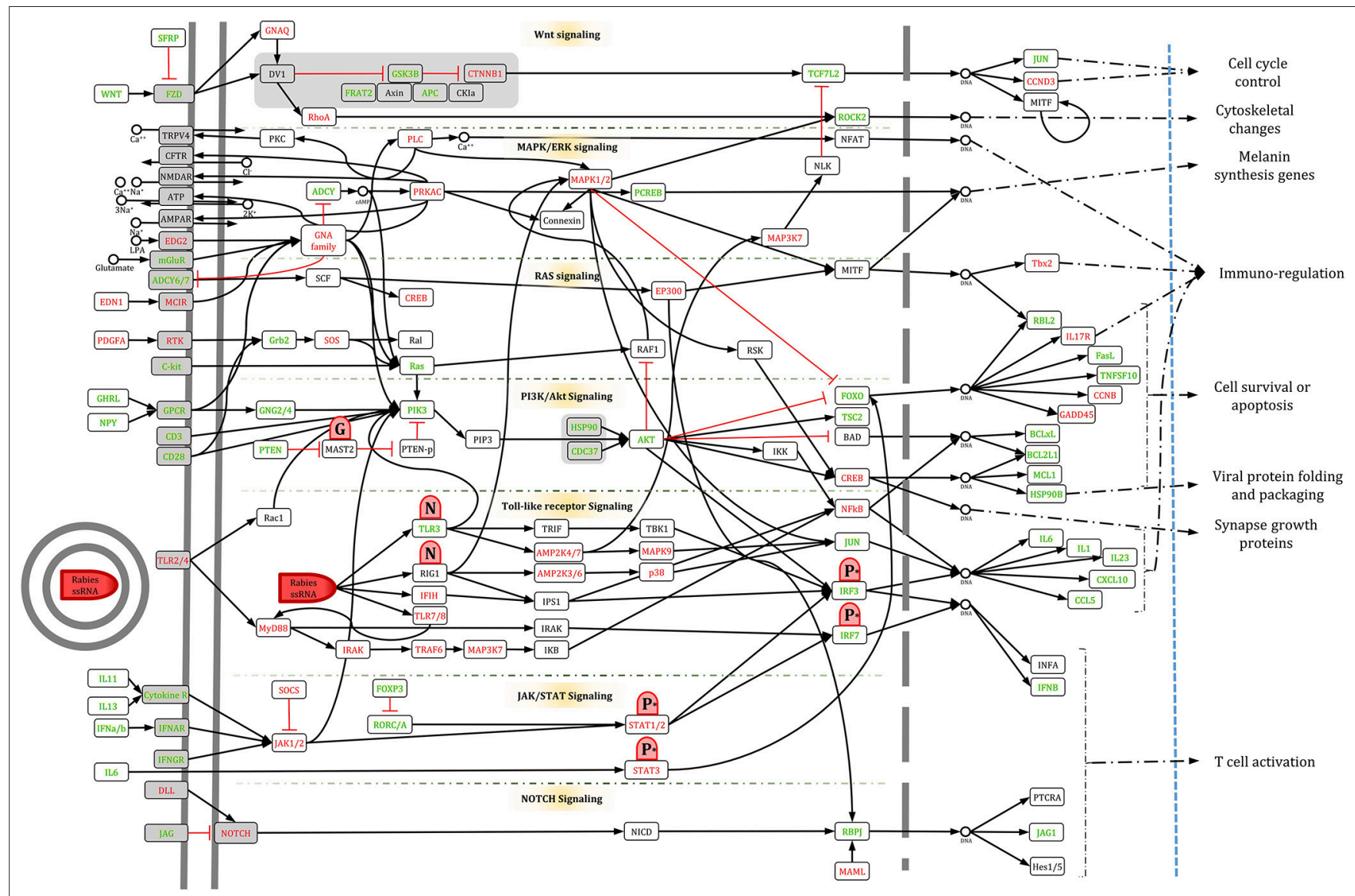

FIGURE 6 | The manually curated version of RISN. The over- and under-expressed genes are colored green and yellow, respectively. The cellular membrane and nuclear membrane are depicted by gray solid and dashed lines, respectively. Proteins are depicted by rounded rectangles, and DNA and small metabolite molecules are shown by circle. The phosphorylated $\mathrm{P}$ protein activated by AKT is denoted by $P^{\star}$, however, for the sake of simplicity, its process is not shown in the signaling pathway. Viral components are depicted by red shapes but with different caps to delineate activatory and inhibitory effects of RABV on RISN.

pathways has a pro-survival function in neurons (Junyent et al., 2010). In a time-course study, Zhao et al. studied the gene expression profile of infected microglial cells and indicated some affected signaling pathways at different time points (Zhao et al., 2013). The MAPK, chemokine, and JAK-STAT signaling were also shown to be implicated in rabies infection along with other innate and adaptive immune response pathways. These pathways were also detected in two other independent studies on CNS of infected mice (Zhao et al., 2011, 2012b).

Viral pattern recognition is critical for early innate immunity response and modulation of pathogen-specific adaptive immunity. TLR3, a member of the TLR family which are pattern recognition receptors in cells, is increased in the cytoplasm of rabies infected cells. It plays major functions in spatial arrangements of infected cells and viral replication, and is observed in endosomes and Negri bodies which are only formed in the presence of TLR3 (Ménager et al., 2009).

NOTCH signaling is important for cell communication, neuronal function, and development in spatial learning and memory (Costa et al., 2003). Our data indicate that this signaling pathway is active in rabid brains. More detailed examination of the role of this signaling pathway in rabies infection is warranted.

\section{Experimental Validation of Expression Alterations}

Gene expression analysis of a number of randomly selected DEGs has been performed previously based on RT-qPCR as a routine validation method of microarray-based expression profiles (Zhao et al., 2011, 2012a,b, 2013) or fluorescent bead immunoassay (Sugiura et al., 2011). In all cases, the former experimentally confirmed DEGs identified in SHIDEGs were STAT1, STAT3, SOCS2, IRF1, IRF3, IRF7, IFNAR2, SH2D1A, CCL3, CCL5, CCRL2, CXCL10, Mx1, IFIT3, OASL2, USP18, IL6, IL10, IL23A, and RTP4. However, we examined another independent random gene set among RISN genes as a further step of validating the microarray-based results. The differential expression of AKT3, GNAI2, and IL21 was analyzed by comparing expression levels in murine neuroblastoma cells infected by the wild type RABV with control uninfected cells using RT-qPCR. The results indicated that the direction of differential expression 
of all three genes were consistent between RT-qPCR results and data integrated from multiple microarray chips (Figure 7 , Supplementary Tables 6, 7). These results confirmed the downregulation of GNAI2 and IL21. AKT3 expression values and statistical tests state that the expression of $A K T 3$ is not upregulated in infected samples. Our findings based on delta $C t$ method and comparing the raw expression values of AKT3 gene in both samples with the referenced values; however, firmly confirm that the value of gene is indeed up-regulated in infected samples.

\section{DISCUSSION}

Rabies is a fatal neuropathological disorder. The fatality of this infection is not because of neurological damage or neurohistopathological signs, but due to neurophysiological disruption of vital signs such as regular heart beat and respiratory rhythm. Other evidences that highlight this neural malfunction are known rabies symptoms such as hydrophobia, photophobia, and paralysis of facial and throat muscles. Although rabies infected cells can mount an innate immune response against this infection, the virus can control the expression and function of the proteins involved in the induction of apoptosis and efficiently suppresses the antiviral innate immune response. From a pathobiological point of view, we acknowledge that the RISN and the previously reported pathways which lead to the spread of RABV can also be triggered by unrelated viruses including other neurotropic RNA viruses, measles, and influenza. This infection would yield similar gene expression profiles by activating general host responses including activation of stress response, innate immune response, and interferon signaling signatures. Based on biological relevance, we partitioned RISN under the following three functional domains.

\section{Interferon Circumvent}

To escape innate and adaptive immunity, rabies perturbs JakStat signaling by influencing interactions and expression. As shown in Figure 5A, two modules of RISN are likely to interfere with this signaling pathway. The inhibition of dimerization of Stat proteins and accumulation in nucleus by viral $\mathrm{P}$ protein are previously described (Vidy et al., 2005; Brzózka et al., 2006; Moseley et al., 2009; Lieu et al., 2013). Our findings showed the

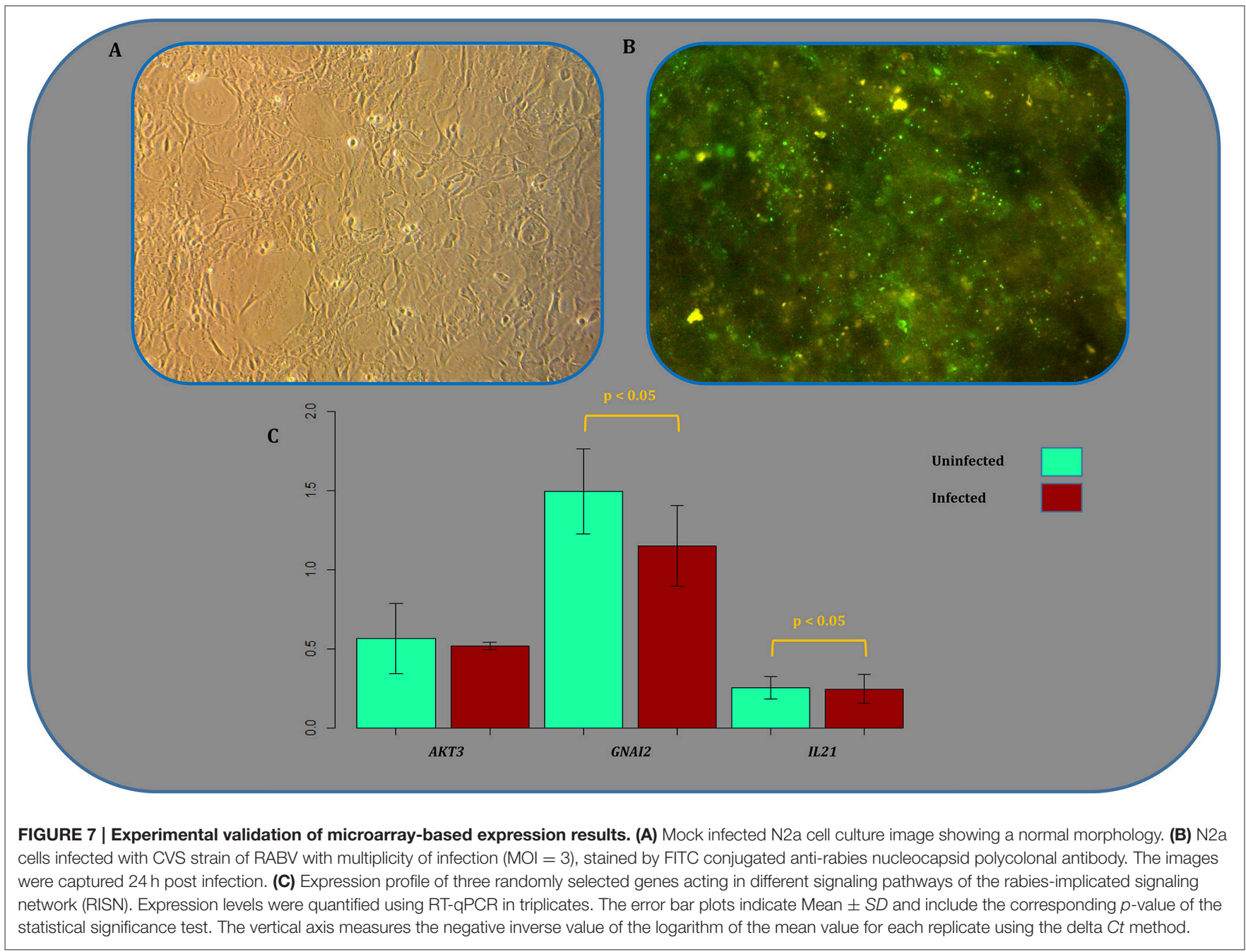


down-regulation of STAT proteins, which is plausible considering the feedback self-loop control on these proteins. This finding is supported by up-regulation of the feedback inhibitor known as the SOCS protein. The high betweenness value of JAK2 and its up-regulation indicate the role of activating innate and adaptive defense systems of infected cells against rabies. Additionally, the low IRI-value of JAK2 and transfer of information toward the "toward proliferation and survival" modules highlight its importance in rabies pathogenesis.

The expression alterations of IL6 and IL21 have been reported in rabies infection previously (Hemachudha et al., 1993; Megid et al., 2006; Quaranta et al., 2008; Dorfmeier et al., 2013; Srithayakumar et al., 2014). The down-regulation of IL21 and its receptors, IL21R, IL15R, and IL17F, following the downregulation of STAT3, is very significant in the observed dampened immune system of the rabid given their role in proliferation and maturation of natural killer cells. Unlikely, the up-regulation of IL6, a neuroprotective cytokine, reinforces the anti-apoptotic effects of the rabies wild type strain. Both of these play a propagation role in this network with IRI-values above one. Despite vastly interfering with the Jak-Stat signaling, rabies infection could not decrease the expression of the famous antiviral molecule, IFNB1, and cell could have upregulated it against the infection. Surprisingly, however, the virus chooses another strategy to skip the interferon mechanism (Faul et al., 2010). This alternative plan is to perturb IFNB1 activation via IRF3. The over-expression of RORA and RORC is also important since they affect the circadian rhythm, calcium-mediated signal transduction and anti-inflammatory responses. It seems that the up-regulated MCL1 and BCL2L1, act in favor of survival, inflammation attenuation and apoptosis inhibition of neurons and disrupt endocytic vesicle retrieval. Also, decrease in function of CIITA and TBX1 causes decrease in the function and efficiency of TH1 and TH2. Overall under-expression of the Notch signaling pathway, including DLL1, NOTCH1, and RBPJ, along with the up-regulation of JAG1, an inhibitor of NOTCH1, is indicative of malfunction in cell-cell communication in CNS and neuronal self-renewal mechanism.

\section{Toward Proliferation and Survival}

Similar to other viral infection, the RABV hijacks the proliferation machine of cells to generate virions as much as possible. To achieve this goal, the strategy of the virus is to keep the cell alive and active and amplifies the production rate. Rabies achieves this by using cell envelope and preventing apoptosis. In fact, there was an inverse correlation between induction of apoptosis and the potency of a virus strain to invade the brain. This suggests that suppression of apoptosis may well be a strategy for neuro-invasiveness of pathogenic RABV and progression through the nervous system (Thoulouze et al., 2003a,b; Larrous et al., 2010). As shown in Figure 5B, up-regulated AKT2 and AKT3 play a central role in the tyrosine kinase module. It has been previously reported that AKT signaling is hijacked by nonsegmented RNA viruses such as vesicular stomatitis virus (VSV) via phosphorylating $\mathrm{P}$ proteins (Sun et al., 2008). It has also been suggested to use AKT inhibitors as an anti-RABVdrug. Our findings underscore the importance of this signaling pathway in neuronal cells where AKT signaling is not normally hyperactive. This activated pathway, prompted by $\mathrm{G}$ viral proteins, may result in the activation of proliferation and growth machinery, and help viral protein folding and packaging via over-expression of HSPs (Lahaye et al., 2009, 2012). TSC2 also triggers apoptosis in immune cells via high representation of FASLG. These results in parallel with those in Sun et al. (2008) highlights the need for studying AKTs and anti-AKTs in rabies models.

Other Serine or Tyrosine kinases, including ITK, IKBKB and RAF1, and PIK3CG were under-expressed, thus resulting in the dampening of the inflammatory response especially with the upregulation of anti-inflammatory proteins such as CHUK and cell proliferatory proteins, NRAS and KRAS. IRF3, IRF7, and IFNB1, which are upregulated naturally in response to viral infection, could not stimulate an immune response due to the activation of the $\mathrm{P}$ viral protein. Besides, IRF3 and IRF7 are involved in AKT activation and transformation of inflammatory to antiinflammatory macrophages (Rieder et al., 2011; Tarassishin et al., 2011). The over-expressed AKT genes also inactivate FOXO3 and therefore disrupt the cell efforts toward apoptosis (Tarassishin et al., 2011). Expression of ANGPTL4 that causes Anoikis, a type of programmed cell death, is also decreased after this module activity (Terada and Nwariaku, 2011). The expression alteration of proteins involved in cell cycle regulation including RB1, RBL1, RBL2, TFDP2, and E2F1 is indicative of the triggering disruption and hijacking by the virus.

\section{Neuropathological Clue}

Hitherto, the underlying mechanism of escape from the immune system, apoptosis prevention and virus production in rabies infection was demonstrated by these modules (Figures 5A,B). However, importantly, the main cause of death in rabid is cardiac arrhythmia and breathing pattern disorders, for which its molecular basis should be tracked elsewhere. Meanwhile, trace of chemokines such as CCL3, CCL5 (a neuron survival factor), and CXCL10 in rabies infection has been previously detected (Nakamichi et al., 2005; Johnson et al., 2008; Li et al., 2012; Huang et al., 2014). These molecules along with their receptors are involved in the blood brain barrier (BBB) permeability and recruitment of different $\mathrm{T}$ cells (Figure 5C). In the natural cell cycle, MYD88 expression leads to an increase in expression of NFKB and hence programmed cell death. Seemingly, the expression and function of MYD88 in addition to NFKB is decreased (Figure 5C) which can lead to the suppression of apoptosis. Diverse chemokines as source nodes in RISN and information transduction to adenylate cyclases and MAP kinases via GNAI2 is likely to be a critical clue to discovering the etiological mechanism of rabies fatality.

Adenylyl cyclases (ADCYs) are central components of signaling cascades downstream of many $G$ proteins. In the mammalians, of the ten ADCY isoforms identified, nine (ADCY1-9) are transmembrane proteins, whereas ADCY10 is a soluble isoform that lacks the transmembrane domains (Sunahara et al., 1996; Conley et al., 2013; Birrell et al., 2015). Although in the RISN the expression of $A D C Y 1$ and $A D C Y 9$ was decreased, the level of ADCY6 and ADCY7 was increased, the overall outcome is probably reduction of signal flow rousted by 
GPCR and ADCY. All ADCY isoforms catalyze the conversion of ATP to cyclic AMP (cAMP) and pyrophosphate. cAMP is a messenger involved in many biological processes including cell growth and differentiation, transcriptional regulation, apoptosis, and various other cellular functions (Patel et al., 2001). The main protein kinase activated by cAMP is protein kinase A (PKA). PKA transfers phosphate groups form ATP to proteins including ion channels on the cell membrane. Similar to changes in enzyme activity following biochemical modification, phosphorylation of ion channel proteins may also cause conformational changes and consequently increase chances of channel opening leading to depolarization of postsynaptic neurons, resulting in firing an action potential and altered electrical activity properties. On the other hand, ADCYs are integrated in lipid rafts and caveolae, and implicated in local cAMP micro-domains in the membrane (Schwencke et al., 1999). Subcellular compartmentalization of protein kinases (such as PKA) and phosphatases, through their interaction with A kinase anchoring protein (AKAPs), provides a mechanism to control signal transduction events at specific sites within the cell (McConnachie et al., 2006). The RABV may interfere with the lipid raft and the micro-compartment associated with the CAMP-AKAP-PKA complex and thus alter ion channel function, eventually leading to neuronal dysfunction. In line with this, it has been reported that NMDA and AMPA glutamate receptors form complexes with cytoskeletal and scaffold proteins in the post-synaptic density (PSD; Kennedy, 1997; Ziff, 1997). Interestingly, AKAP binds to PSD in complexes with NMDA and AMPA receptors (Colledge et al., 2000). It is also thought that regulation of this molecular architecture is essential for controlling glutamate receptors in hippocampal long-term potentiation (LTP) and long-term depression (LTD) synaptic plasticity (Lüscher et al., 2000; Tomita et al., 2001). Our data showed that the expression of PRKACA and AKAP13 subsequent to GNAI2 decreased significantly (Supplementary Table 5). It therefore seems that these complexes may be a preferential target of viruses to hijack cellular machinery.

Neuropathological observations indicate that functional alterations precede neuronal death, which is responsible for the clinical manifestation and fatal outcome in rabies. Indeed, Gourmelon et al. reported that disappearance of rapid eye movement (REM) sleep and the development of pseudoperiodic facial myoclonus are the first manifestations in the EEG recordings of mice infected with the challenge virus standard (CVS) of fixed RV (Gourmelon et al., 1986). It has also been reported that electrical activity of brain terminates $30 \mathrm{~min}$ prior to the cardiac arrest, indicating that cerebral death occurs before vegetative function failure in experimental rabies (Fu and Jackson, 2005). Considering the increased activity of voltage-gated channels by phosphorylation in response to PKA stimulation, initiating a signaling pathway from ADCY to ion channel functioning could be a possible mechanism by which the RABV hijacks the neurons. Consistently, Iwata et al. showed that ion channel dysfunction occurs in mouse neuroblastoma cells infected by RV (Iwata et al., 1999). They reported that not only the functional activity of voltage-dependent sodium and inward rectifier potassium channels were decreased, but also the resting membrane potential was decreased, indicating membrane depolarization. Therefore, decreased activity of these channels could preclude infected neurons from firing action potentials and generating synaptic potentials, thus leading to functional impairment. Fu and Jackson (2005) observed that neurotransmitter releases from rat hippocampus, after inoculation with RV CVS-24, was increased at day 1, reached a peak at day 3 , and then declined by day 5 . Manifestations of clinical signs of rabies were consistent with day 5 of inoculation when neurotransmitter release was equal or below the level prior to infection, suggesting that neurons are no longer capable of releasing neurotransmitters at the synaptic junctions and this may be the underlying basis of clinical signs including paralysis (Fu and Jackson, 2005).

Since there is a paucity of data pertaining to rabies influence on physiological processes, particularly on neuronal electrophysiological properties, further studies need to be undertaken to confirm whether neuronal dysfunction occurring in rabies infection is due to an aberrant signaling pathway initiating from ADCY-cAMP-PKA and finalizing with ion channel phosphorylation. It should be noted that any alterations in different ion channels may result in dysfunction of neurons and brain regions which are responsible for vital tasks including attention, thinking and respiration.

\section{CONCLUSION}

Knowledge-driven studies are mostly non-automatic, heuristic, expert-dependent, and evidence-based surveys. Although this strategy of problem solving is valuable in identification of novel findings, it suffers from certain limitations and subjective biases. With the emergence of omics technologies, data-driven studies have exploited the large and ever-growing publicly available deposited datasets as a complementary approach to knowledgebased studies (Sun et al., 2012). Data-driven approaches are computationally demanding and require complex interpretations but this is dependent directly to the original data itself (Hua et al., 2006; Sun et al., 2012). Here, we combined data- and knowledge-driven studies to potentially identify a less-biased signaling network of rabies infection. We thus undertook a systematic approach which initiated with a data-driven approach and was then extended by a comprehensive complementary knowledge-based approach. In addition, we included all available high-throughput whole-transcriptome datasets in a horizontal (meta-analysis) and super-horizontal (miRNA and mRNA) integration approach. Critically, signaling pathways were then used as a scaffold for data integration to identify key players in signaling pathways and genes. Finally, we constructed a bird's-eye view map, RISN, of signaling deviations including host-pathogen interaction data. Uniquely, this signaling pathway illustrates the host-rabies interaction signature.

In summary, we demonstrate that seven signaling pathways including (1) WNT, (2) MAPK/ERK, (3) RAS, (4) PI3K/AKT, (5) Toll-like receptor, (6) JAK/STAT, and (7) NOTCH are involved in controlling cell cycle, cell survival, viral replication and folding, synapse regulation, and regulation of immunity. Among the many involved proteins, divergence and convergence 
of signals indicates that PLC, MAPK1/2, PIK3, PKC, and JAK are potentially the most critical of all in rabies pathogenesis. Interestingly, signals are converged toward these proteins and are then diverged toward several distinct transcription factors and end-point biological processes (Figure 8).

In addition to confirming former reports on the inhibition of apoptosis in neurons, RISN provided molecular evidence of interferon escape and neural cell death prevention in rabies infection. This finding is significant given that it explicates how the virus continues to parasitically multiply without any neural host cell damage. Data herein suggest that, the RABV hijacks the phosphorylation machinery of the cell to facilitate its own replication. Also, the tight regulation of recruited immune cells by the virus is demonstrated. The network analysis also

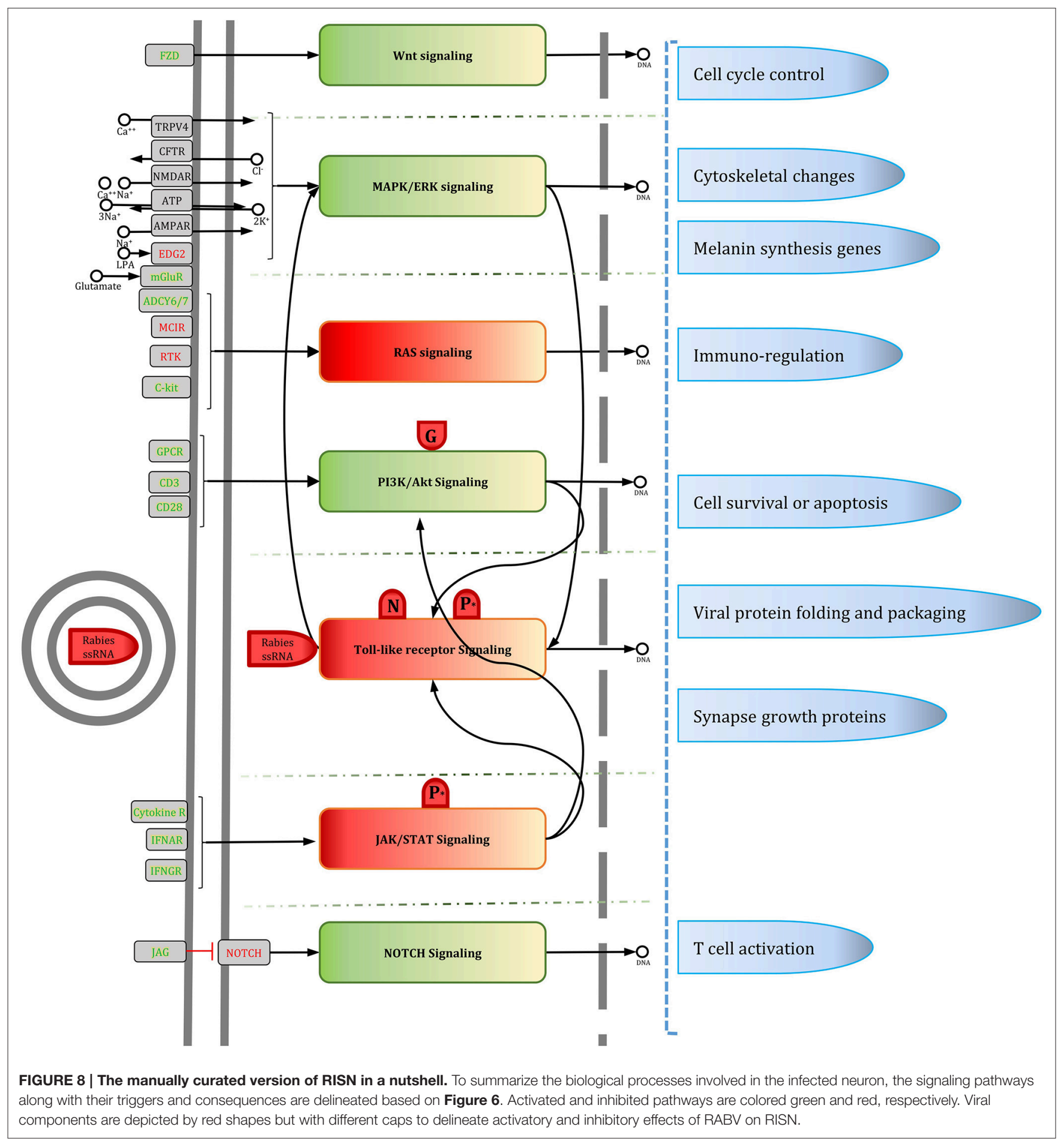


shed light on the gene set central to rabies infection, all of which were bottlenecks in RISN. Moreover, based on RISN, we hypothesize that modifying certain signal transduction apparatus involved in rabies pathogenesis such as the cAMP or AKT signaling pathway may instigate an effective immune response which will consequently diminish the fatality of the rabies infection.

The systems biomedicine approach employed in this study provided a better understanding of the underlying signaling network of this infectious disease. Further independent validation of the RISN potentially provides a molecular framework for intervention and development of novel effective treatments for the late stages of this neglected disease.

\section{AUTHOR CONTRIBUTIONS}

MJ conducted the design of study and carried out literature review, data collection, data analysis, and implemented the computational methods. SHM did the literature search and some analysis. MJ, SAJ, and HP participated in network analysis. AG and NA performed the experimental validation. MJ, NA, AG, $\mathrm{SAJ}$ and SHM wrote the paper. MM, FN, and BV participated in revising the manuscript critically. All authors read and approved the final manuscript.

\section{FUNDING}

This work was supported with a research grant received from Pasteur Institute of Iran (No. 748).

\section{REFERENCES}

Amiri, M., Jafari, M., Azimzadeh Jamalkandi, S., and Davoodi, S.-M. (2013). Atopic dermatitis-associated protein interaction network lead to new insights in chronic sulfur mustard skin lesion mechanisms. Expert Rev. Proteomics 10, 449-460. doi: 10.1586/14789450.2013.841548

Ansari-Pour, N., Razaghi-Moghadam, Z., Barneh, F., and Jafari, M. (2016). Testisspecific Y-centric protein-protein interaction network provides clues to the etiology of severe spermatogenic failure. J. Proteome Res. 15, 1011-1022. doi: 10.1021/acs.jproteome.5b01080

Azimzadeh Jamalkandi, S., Mirzaie, M., Jafari, M., Mehrani, H., Shariati, P., and Khodabandeh, M. (2015). Signaling network of lipids as a comprehensive scaffold for omics data integration in sputum of COPD patients. Biochim. Biophys. Acta 1851, 1383-1393. doi: 10.1016/j.bbalip.2015. 07.005

Baloul, L., Camelo, S., and Lafon, M. (2004). Up-regulation of Fas ligand (FasL) in the central nervous system: a mechanism of immune evasion by rabies virus. $J$. Neurovirol. 10, 372-382. doi: 10.1080/13550280490521122

Baloul, L., and Lafon, M. (2003). Apoptosis and rabies virus neuroinvasion. Biochimie 85, 777-788. doi: 10.1016/S0300-9084(03)00137-8

Bastian, M., Heymann, S., and Jacomy, M. (2009). "Gephi: an open source software for exploring and manipulating networks," in International AAAI Conference on Weblogs and Social Media.

Bender, R. H. F., Haigis, K. M., and Gutmann, D. H. (2015). Activated k-ras, but not h-ras or N-ras, regulates brain neural stem cell proliferation in a raf/rb-dependent manner. Stem Cells 33, 1998-2010. doi: 10.1002/stem.1990

Bindea, G., Mlecnik, B., Hackl, H., Charoentong, P., Tosolini, M., Kirilovsky, A., et al. (2009). ClueGO: a Cytoscape plug-in to decipher functionally

\section{ACKNOWLEDGMENTS}

The authors wish to thank Karl-Klaus Conzelmann, Monique Lafon, and Hervé Bourhy for providing us with in-depth knowledge on rabies and their critical evaluation of the manuscript.

\section{SUPPLEMENTARY MATERIAL}

The Supplementary Material for this article can be found online at: http://journal.frontiersin.org/article/10.3389/fmicb. 2016.01688/full\#supplementary-material

Supplementary Table 1 | The selected datasets for rabies data integration.

Supplementary Table 2 | The gene ontology enrichment analysis of differentially expressed and central (degree and betweenness) genes in SHIDEG-PPIN.

Supplementary Table 3 | The full results of pathway enrichment analysis of SHIDEG-PPIN modules by ClueGO.

Supplementary Table 4 | The KEGG enriched pathway similarity matrix and the module interconnectivity matrix of RISN.

Supplementary Table 5 | Properties of RISN nodes and list of all edges.

Supplementary Table 6 | The over- and under-expression of 694 DEGs in the unrefined RISN.

Supplementary Table 7 | Properties of refined SHIDEG-PPIN nodes and list of edges.

Supplementary Figure 1 | The degree distribution of the refined SHIDEG-PPIN.

Data Sheet 1 | The computational scripts plus an example of raw data used in this study.

grouped gene ontology and pathway annotation networks. Bioinformatics 25, 1091-1093. doi: 10.1093/bioinformatics/btp101

Birrell, M. A., Bonvini, S. J., Wortley, M. A., Buckley, J., Yew-Booth, L., Maher, S. A., et al. (2015). The role of adenylyl cyclase isoform 6 in betaadrenoceptor signalling in murine airways. Br. J. Pharmacol. 172, 131-141. doi: 10.1111/bph.12905

Bowick, G. C., and McAuley, A. J. (2011). Meta-analysis of high-throughput datasets reveals cellular responses following hemorrhagic fever virus infection. Viruses 3, 613-619. doi: 10.3390/v3050613

Brzózka, K., Finke, S., and Conzelmann, K. K. (2006). Inhibition of interferon signaling by rabies virus phosphoprotein P: activation-dependent binding of STAT1 and STAT2. J. Virol. 80, 2675-2683. doi: 10.1128/JVI.80.6.26752683.2006

Camacho-Cáceres, K. I., Acevedo-Díaz, J. C., Pérez-Marty, L. M., Ortiz, M., Irizarry, J., Cabrera-Ríos, M., et al. (2015). Multiple criteria optimization joint analyses of microarray experiments in lung cancer: from existing microarray data to new knowledge. Cancer Med. 4, 1884-1900. doi: 10.1002/cam4.540

Ceccaldi, P. E., Valtorta, F., Braud, S., Hellio, R., and Tsiang, H. (1997). Alteration of the actin-based cytoskeleton by rabies virus. J. Gen. Virol. 78(Pt 11), 2831-2835. doi: 10.1099/0022-1317-78-11-2831

Chen, X., Liang, S., Zheng, W., Liao, Z., Shang, T., and Ma, W. (2008). Metaanalysis of nasopharyngeal carcinoma microarray data explores mechanism of EBV-regulated neoplastic transformation. BMC Genomics 9:322. doi: 10.1186/1471-2164-9-322

Chen, E. Y., Tan, C. M., Kou, Y., Duan, Q., Wang, Z., Meirelles, G. V., et al. (2013). Enrichr: interactive and collaborative HTML5 gene list enrichment analysis tool. BMC Bioinformatics 14:128. doi: 10.1186/1471-2105$14-128$ 
Colledge, M., Dean, R. A., Scott, G. K., Langeberg, L. K., Huganir, R. L., and Scott, J. D. (2000). Targeting of PKA to glutamate receptors through a MAGUK-AKAP complex. Neuron 27, 107-119. doi: 10.1016/S0896-6273(00)00013-1

Conley, J. M., Brand, C. S., Bogard, A. S., Pratt, E. P., Xu, R., Hockerman, G. H., et al. (2013). Development of a high-throughput screening paradigm for the discovery of small-molecule modulators of adenylyl cyclase: identification of an adenylyl cyclase 2 inhibitor. J. Pharmacol. Exp. Ther. 347, 276-287. doi: 10.1124/jpet.113.207449

Costa, R. M., Honjo, T., and Silva, A. J. (2003). Learning and memory deficits in Notch mutant mice. Curr. Biol. 13, 1348-1354. doi: 10.1016/S09609822(03)00492-5

Croft, D., Mundo, A. F., Haw, R., Milacic, M., Weiser, J., Wu, G., et al. (2014). The Reactome pathway knowledgebase. Nucleic Acids Res. 42, D472-D477. doi: 10.1093/nar/gkt1102

Csardi, G., and Nepusz, T. (2006). The igraph software package for complex network research. InterJournal Compl. Syst. 1695.

Depiereux, S., Le Gac, F., De Meulder, B., Pierre, M., Helaers, R., Guiguen, Y., et al. (2015). Meta-analysis of microarray data of rainbow trout fry gonad differentiation modulated by ethynylestradiol. PLOS ONE 10:e0135799. doi: 10.1371/journal.pone.0135799

de Souza, A., and Madhusudana, S. N. (2014). Survival from rabies encephalitis. J. Neurol. Sci. 339, 8-14. doi: 10.1016/j.jns.2014.02.013

Dhingra, V., Li, X., Liu, Y., and Fu, Z. F. (2007). Proteomic profiling reveals that rabies virus infection results in differential expression of host proteins involved in ion homeostasis and synaptic physiology in the central nervous system. J. Neurovirol. 13, 107-117. doi: 10.1080/13550280601178226

Dorfmeier, C. L., Tzvetkov, E. P., Gatt, A., and McGettigan, J. P. (2013). Investigating the role for IL-21 in rabies virus vaccine-induced immunity. PLoS Negl. Trop. Dis. 7:e2129. doi: 10.1371/journal.pntd.0002129

Farahtaj, F., Zandi, F., Khalaj, V., Biglari, P., Fayaz, A., and Vaziri, B. (2013). Proteomics analysis of human brain tissue infected by street rabies virus. Mol. Biol. Rep. 40, 6443-6450. doi: 10.1007/s11033-013-2759-0

Faul, E. J., Wanjalla, C. N., Suthar, M. S., Gale, M., Wirblich, C., and Schnell, M. J. (2010). Rabies virus infection induces type I interferon production in an IPS1 dependent manner while dendritic cell activation relies on IFNAR signaling. PLoS Pathog. 6:e1001016. doi: 10.1371/journal.ppat.1001016

Francischetti, I. M., Assumpção, T. C., Ma, D., Li, Y., Vicente, E. C., Uieda, W., et al. (2013). The "Vampirome": transcriptome and proteome analysis of the principal and accessory submaxillary glands of the vampire bat Desmodus rotundus, a vector of human rabies. J. Proteomics 82, 288-319. doi: 10.1016/j.jprot.2013.01.009

Fu, Z. F., and Jackson, A. C. (2005). Neuronal dysfunction and death in rabies virus infection. J. Neurovirol. 11, 101-106. doi: 10.1080/13550280590900445

Fu, Z. F., Li, X., and Dhingra, V. (2008). Pathogenic rabies virus alters host protein expression in the central nervous system: implications for neuronal dysfunction. Dev. Biol. (Basel). 131, 83-91.

Galelli, A., Baloul, L., and Lafon, M. (2000). Abortive rabies virus central nervous infection is controlled by $\mathrm{T}$ lymphocyte local recruitment and induction of apoptosis. J. Neurovirol. 6, 359-372. doi: 10.3109/13550280009018300

Gautier, L., Cope, L., Bolstad, B. M., and Irizarry, R. A. (2004). Affy-analysis of Affymetrix GeneChip data at the probe level. Bioinformatics 20, 307-315. doi: 10.1093/bioinformatics/btg405

Gitter, A., Klein-Seetharaman, J., Gupta, A., and Bar-Joseph, Z. (2011). Discovering pathways by orienting edges in protein interaction networks. Nucleic Acids Res. 39, e22. doi: 10.1093/nar/gkq1207

Gomme, E. A., Wirblich, C., Addya, S., Rall, G. F., and Schnell, M. J. (2012). Immune clearance of attenuated rabies virus results in neuronal survival with altered gene expression. PLoS Pathog. 8:e1002971. doi: 10.1371/journal.ppat.1002971

Good, P. F., Olanow, C. W., and Perl, D. P. (1992). Neuromelanin-containing neurons of the substantia nigra accumulate iron and aluminum in Parkinson's disease: a LAMMA study. Brain Res. 593, 343-346. doi: 10.1016/00068993(92)91334-B

Gourmelon, P., Briet, D., Court, L., and Tsiang, H. (1986). Electrophysiological and sleep alterations in experimental mouse rabies. Brain Res. 398, 128-140. doi: 10.1016/0006-8993(86)91258-8

Han, M. G., Park, J. S., Lee, C. S., Jeong, Y. E., Ryou, J. S., Cho, J. E., et al. (2011). Serum microRNA expression profiling in mice infected with rabies virus. Osong. Public Health Res. Perspect. 2, 186-191. doi: 10.1016/j.phrp.2011. 11.043

Hardin, J., and Wilson, J. (2009). A note on oligonucleotide expression values not being normally distributed. Biostatistics 10, 446-450. doi: 10.1093/biostatistics/kxp003

Hemachudha, T., Panpanich, T., Phanuphak, P., Manatsathit, S., and Wilde, H. (1993). Immune activation in human rabies. Trans. R. Soc. Trop. Med. Hyg. 87, 106-108. doi: 10.1016/0035-9203(93)90446-W

Hua, F., Hautaniemi, S., Yokoo, R., and Lauffenburger, D. A. (2006). Integrated mechanistic and data-driven modelling for multivariate analysis of signalling pathways. J. R. Soc. Interface 3, 515-526. doi: 10.1098/rsif.2005.0109

Huang, Y., Jiao, S., Tao, X., Tang, Q., Jiao, W., Xiao, J., et al. (2014). Met-CCL5 represents an immunotherapy strategy to ameliorate rabies virus infection. $J$. Neuroinflammation 11:146. doi: 10.1186/s12974-014-0146-y

Hunter, D. J. (2005). Gene-environment interactions in human diseases. Nat. Rev. Genet. 6, 287-298. doi: 10.1038/nrg1578

Ito, N., Mita, T., Shimizu, K., Ito, Y., Masatani, T., Nakagawa, K., et al. (2011). Amino acid substitution at position 95 in rabies virus matrix protein affects viral pathogenicity. J. Vet. Med. Sci. 73, 1363-1366. doi: 10.1292/jvms.11-0151

Iwata, M., Komori, S., Unno, T., Minamoto, N., and Ohashi, H. (1999). Modification of membrane currents in mouse neuroblastoma cells following infection with rabies virus. Br. J. Pharmacol. 126, 1691-1698. doi: 10.1038/sj.bjp.0702473

Jafari, M., Mirzaie, M., and Sadeghi, M. (2015). Interlog protein network: an evolutionary benchmark of protein interaction networks for the evaluation of clustering algorithms. BMC Bioinformatics 16:319. doi: 10.1186/s12859-0150755-1

Jafari, M., Sadeghi, M., Mirzaie, M., Marashi, S.-A., and Rezaei-Tavirani, M. (2013). Evolutionarily conserved motifs and modules in mitochondrial protein-protein interaction networks. Mitochondrion 13, 668-675. doi: 10.1016/j.mito.2013.09.006

Johnson, N., Mansfield, K. L., Hicks, D., Nunez, A., Healy, D. M., Brookes, S. M. et al. (2008). Inflammatory responses in the nervous system of mice infected with a street isolate of rabies virus. Dev. Biol. (Basel). 131, 65-72.

Junyent, F., Alvira, D., Yeste-Velasco, M., de la Torre, A. V., Beas-Zarate, C., Sureda, F. X., et al. (2010). Prosurvival role of JAK/STAT and Akt signaling pathways in MPP+-induced apoptosis in neurons. Neurochem. Int. 57, 774-782. doi: 10.1016/j.neuint.2010.08.015

Kammouni, W., Wood, H., Saleh, A., Appolinario, C. M., Fernyhough, P., and Jackson, A. C. (2015). Rabies virus phosphoprotein interacts with mitochondrial Complex I and induces mitochondrial dysfunction and oxidative stress. J. Neurovirol. 21, 370-382. doi: 10.1007/s13365-0150320-8

Kanehisa, M., Goto, S., Sato, Y., Kawashima, M., Furumichi, M., and Tanabe, M. (2014). Data, information, knowledge and principle: back to metabolism in KEGG. Nucleic Acids Res. 42, D199-D205. doi: 10.1093/nar/gkt1076

Kasempimolporn, S., Lumlertdacha, B., Chulasugandha, P., Boonchang, S., and Sitprija, V. (2014). Alterations in brain cerebral cortex proteome of rabiesinfected cat. Southeast Asian J. Trop. Med. Public Health 45, 808-815.

Kelder, T., van Iersel, M. P., Hanspers, K., Kutmon, M., Conklin, B. R., Evelo, C. T., et al. (2012). WikiPathways: building research communities on biological pathways. Nucl. Acids Res. 40, D1301-D1307. doi: 10.1093/nar/gkr1074

Kennedy, M. B. (1997). The postsynaptic density at glutamatergic synapses. Trends Neurosci. 20, 264-268. doi: 10.1016/S0166-2236(96)01033-8

Kluge, S., Rourou, S., Vester, D., Majoul, S., Benndorf, D., Genzel, Y., et al. (2013). Proteome analysis of virus-host cell interaction: rabies virus replication in Vero cells in two different media. Appl. Microbiol. Biotechnol. 97, 5493-5506. doi: 10.1007/s00253-013-4939-1

Kuleshov, M. V., Jones, M. R., Rouillard, A. D., Fernandez, N. F., Duan, Q., Wang, Z., et al. (2016). Enrichr: a comprehensive gene set enrichment analysis web server 2016 update. Nucl. Acids Res., 44, W90-W97. doi: 10.1093/nar/gkw377

Koschel, K., and Halbach, M. (1979). Rabies virus infection selectively impairs membrane receptor functions in neuronal model cells. J. Gen. Virol. 42, 627-632. doi: 10.1099/0022-1317-42-3-627

Koschel, K., and Münzel, P. (1984). Inhibition of opiate receptor-mediated signal transmission by rabies virus in persistently infected NG-108-15 mouse neuroblastoma-rat glioma hybrid cells. Proc. Natl. Acad. Sci. U.S.A. 81, 950-954. doi: 10.1073/pnas.81.3.950 
Lahaye, X., Vidy, A., Fouquet, B., and Blondel, D. (2012). Hsp70 protein positively regulates rabies virus infection. J. Virol. 86, 4743-4751. doi: 10.1128/JVI. 06501-11

Lahaye, X., Vidy, A., Pomier, C., Obiang, L., Harper, F., Gaudin, Y., et al. (2009). Functional characterization of Negri bodies (NBs) in rabies virus-infected cells: evidence that NBs are sites of viral transcription and replication. J. Virol. 83, 7948-7958. doi: 10.1128/JVI.00554-09

Langfelder, P., and Horvath, S. (2008). WGCNA: an R package for weighted correlation network analysis. BMC Bioinformatics 9:559. doi: 10.1186/14712105-9-559

Larrous, F., Gholami, A., Mouhamad, S., Estaquier, J., and Bourhy, H. (2010). Two overlapping domains of a lyssavirus matrix protein that acts on different cell death pathways. J. Virol. 84, 9897-9906. doi: 10.1128/JVI.00761-10

Lay, S., Préhaud, C., Dietzschold, B., and Lafon, M. (2003). Glycoprotein of nonpathogenic rabies viruses is a major inducer of apoptosis in human jurkat T cells. Ann. N. Y. Acad. Sci. 1010, 577-581. doi: 10.1196/annals.1299.108

Li, A., and Horvath, S. (2007). Network neighborhood analysis with the multi-node topological overlap measure. Bioinformatics 23, 222-231. doi: 10.1093/bioinformatics/btl581

Li, J., Ertel, A., Portocarrero, C., Barkhouse, D. A., Dietzschold, B., Hooper, D. C., et al. (2012). Postexposure treatment with the live-attenuated rabies virus (RV) vaccine TriGAS triggers the clearance of wild-type RV from the Central Nervous System (CNS) through the rapid induction of genes relevant to adaptive immunity in CNS tissues. J. Virol. 86, 3200-3210. doi: 10.1128/JVI.06699-11

Lieu, K. G., Brice, A., Wiltzer, L., Hirst, B., Jans, D. A., Blondel, D., et al. (2013). The rabies virus interferon antagonist $P$ protein interacts with activated STAT3 and inhibits Gp130 receptor signaling. J. Virol. 87, 8261-8265. doi: 10.1128/JVI.00989-13

Livak, K. J., and Schmittgen, T. D. (2001). Analysis of relative gene expression data using real-time quantitative PCR and the $2^{-\Delta \Delta C T}$ Method. Methods 25, 402-408. doi: 10.1006/meth.2001.1262

Lüscher, C., Nicoll, R. A., Malenka, R. C., and Muller, D. (2000). Synaptic plasticity and dynamic modulation of the postsynaptic membrane. Nat. Neurosci. 3, 545-550. doi: 10.1038/75714

McConnachie, G., Langeberg, L. K., and Scott, J. D. (2006). AKAP signaling complexes: getting to the heart of the matter. Trends Mol. Med. 12, 317-323. doi: 10.1016/j.molmed.2006.05.008

Megid, J., Appolinario, C. M., Mazzini, A. M., and Almeida, M. F. (2006). Evaluation of cytokines concentration and percentage of survival of rabies virus-infected mice submitted to anti-rabies Vero-cell propagated vaccine and P. acnes. Vet. Immunol. Immunopathol. 114, 192-196. doi: 10.1016/j.vetimm.2006.07.010

Mehta, S. M., Banerjee, S. M., and Chowdhary, A. S. (2015). Postgenomics biomarkers for rabies-the next decade of proteomics. OMICS 19, 67-79. doi: 10.1089/omi.2014.0127

Ménager, P., Roux, P., Mégret, F., Bourgeois, J.-P., Le Sourd, A.-M., Danckaert, A., et al. (2009). Toll-like receptor 3 (TLR3) plays a major role in the formation of rabies virus Negri Bodies. PLoS Pathog. 5:e1000315. doi: 10.1371/journal.ppat.1000315

Meslin, F. X., Kaplan, M. M., and Koprowski, H. (1996). Laboratory Technique in Rabies. World Health Organization. Geneva: World Health Organization.

Moseley, G. W., Lahaye, X., Roth, D. M., Oksayan, S., Filmer, R. P., Rowe, C. L., et al. (2009). Dual modes of rabies P-protein association with microtubules: a novel strategy to suppress the antiviral response. J. Cell Sci. 122(Pt 20), 3652-3662. doi: $10.1242 /$ jcs. 045542

Mundschau, L. J., and Faller, D. V. (1994). Endogenous inhibitors of the dsRNAdependent eIF-2 alpha protein kinase PKR in normal and ras-transformed cells. Biochimie 76, 792-800. doi: 10.1016/0300-9084(94)90083-3

Nakamichi, K., Inoue, S., Takasaki, T., Morimoto, K., and Kurane, I. (2004). Rabies virus stimulates nitric oxide production and CXC chemokine ligand 10 expression in macrophages through activation of extracellular signalregulated kinases 1 and 2. J. Virol. 78, 9376-9388. doi: 10.1128/JVI.78.17.93769388.2004

Nakamichi, K., Saiki, M., Sawada, M., Takayama-Ito, M., Yamamuro, Y., Morimoto, K., et al. (2005). Rabies virus-induced activation of mitogenactivated protein kinase and NF-kappaB signaling pathways regulates expression of CXC and CC chemokine ligands in microglia. J. Virol. 79, 11801-11812. doi: 10.1128/JVI.79.18.11801-11812.2005

Paraboschi, E., Cardamone, G., Rimoldi, V., Gemmati, D., Spreafico, M., Duga, S., et al. (2015). Meta-analysis of multiple sclerosis microarray data reveals dysregulation in RNA splicing regulatory genes. Int. J. Mol. Sci. 16, 23463-23481. doi: 10.3390/ijms161023463

Patel, T. B., Du, Z., Pierre, S., Cartin, L., and Scholich, K. (2001). Molecular biological approaches to unravel adenylyl cyclase signaling and function. Gene 269, 13-25. doi: 10.1016/S0378-1119(01)00448-6

Préhaud, C., Lay, S., Dietzschold, B., and Lafon, M. (2003). Glycoprotein of nonpathogenic rabies viruses is a key determinant of human cell apoptosis. J. Virol. 77, 10537-10547. doi: 10.1128/JVI.77.19.10537-10547.2003

Quaranta, A., Siniscalchi, M., Albrizio, M., Volpe, S., Buonavoglia, C., and Vallortigara, G. (2008). Influence of behavioural lateralization on interleukin-2 and interleukin- 6 gene expression in dogs before and after immunization with rabies vaccine. Behav. Brain Res. 186, 256-260. doi: 10.1016/j.bbr.2007.08.014

Ramasamy, A., Mondry, A., Holmes, C. C., and Altman, D. G. (2008). Key issues in conducting a meta-analysis of gene expression microarray datasets. PLoS Med. 5:e184. doi: 10.1371/journal.pmed.0050184

Rieder, M., Brzózka, K., Pfaller, C. K., Cox, J. H., Stitz, L., and Conzelmann, K. K. (2011). Genetic dissection of interferon-antagonistic functions of rabies virus phosphoprotein: inhibition of interferon regulatory factor 3 activation is important for pathogenicity. J. Virol. 85, 842-852. doi: 10.1128/JVI.01427-10

Rupprecht, C. E. (1996). "Rhabdoviruses: rabies virus," in Medical Microbiology, 4th Edn., ed S. Baron (Galveston, TX: University of Texas Medical Branch at Galveston).

Russell, S. J. (2002). RNA viruses as virotherapy agents. Cancer Gene Ther. 9, 961-966. doi: 10.1038/sj.cgt.7700535

Sagara, J., Tsukita, S., Yonemura, S., Tsukita, S., and Kawai, A. (1995). Cellular actin-binding ezrin-radixin-moesin (ERM) family proteins are incorporated into the rabies virion and closely associated with viral envelope proteins in the cell. Virology 206, 485-494. doi: 10.1016/S0042-6822(95)80064-6

Sarmento, L., Tseggai, T., Dhingra, V., and Fu, Z. F. (2006). Rabies virus-induced apoptosis involves caspase-dependent and caspase-independent pathways. Virus Res. 121, 144-151. doi: 10.1016/j.virusres.2006.05.002

Schnell, M. J., McGettigan, J. P., Wirblich, C., and Papaneri, A. (2010). The cell biology of rabies virus: using stealth to reach the brain. Nat. Rev. Microbiol. 8, 51-61. doi: 10.1038/nrmicro2260

Schwencke, C., Yamamoto, M., Okumura, S., Toya, Y., Kim, S. J., and Ishikawa, Y. (1999). Compartmentation of cyclic adenosine $3^{\prime}, 5^{\prime}$-monophosphate signaling in caveolae. Mol. Endocrinol. 13, 1061-1070. doi: 10.1210/mend.13.7.0304

Sharma, M., Ying, R., Tarr, G., and Barnabas, R. (2015). Systematic review and meta-analysis of community and facility-based HIV testing to address linkage to care gaps in sub-Saharan Africa. Nature 528, S77-S85. doi: 10.1038 /nature 16044

Silva, S. R., Katz, I. S., Mori, E., Carnieli, P. Jr., Vieira, L. F., Batista, H. B., et al. (2013). Biotechnology advances: a perspective on the diagnosis and research of Rabies Virus. Biologicals 41, 217-223. doi: 10.1016/j.biologicals.2013.04.002

Silverbush, D., and Sharan, R. (2014). Network orientation via shortest paths. Bioinformatics 30, 1449-1455. doi: 10.1093/bioinformatics/btu043

Song, G. G., Kim, J.-H., Seo, Y. H., Choi, S. J., Ji, J. D., and Lee, Y. H. (2014). Meta-analysis of differentially expressed genes in primary Sjogren's syndrome by using microarray. Hum. Immunol. 75, 98-104. doi: 10.1016/j.humimm.2013.09.012

Song, L., Langfelder, P., and Horvath, S. (2012). Comparison of co-expression measures: mutual information, correlation, and model based indices. BMC Bioinformatics 13:328. doi: 10.1186/1471-2105-13-328

Song, Y., Hou, J., Qiao, B., Li, Y., Xu, Y., Duan, M., et al. (2013). Street rabies virus causes dendritic injury and F-actin depolymerization in the hippocampus. $J$. Gen. Virol. 94(Pt 2), 276-283. doi: 10.1099/vir.0.047480-0

Srithayakumar, V., Sribalachandran, H., Rosatte, R., Nadin-Davis, S. A., and Kyle, C. J. (2014). Innate immune responses in raccoons after raccoon rabies virus infection. J. Gen. Virol. 95(Pt 1), 16-25. doi: 10.1099/vir.0.053942-0

Sugiura, N., Uda, A., Inoue, S., Kojima, D., Hamamoto, N., Kaku, Y., et al. (2011). Gene expression analysis of host innate immune responses in the central nervous system following lethal CVS-11 infection in mice. Jpn. J. Infect. Dis. $64,463-472$. 
Sun, J., Hu, J., Luo, D., Markatou, M., Wang, F., Edabollahi, S., et al. (2012). "Combining knowledge and data driven insights for identifying risk factors using electronic health records," in Annual Symposium Proceedings/AMIA Symposium (Chicago, IL), 901-910. Available online at: https://www.amia.org/ amia2012

Sun, M., Fuentes, S. M., Timani, K., Sun, D., Murphy, C., Lin, Y., et al. (2008). Akt plays a critical role in replication of nonsegmented negative-stranded RNA viruses. J. Virol. 82, 105-114. doi: 10.1128/JVI.01 520-07

Sunahara, R. K., Dessauer, C. W., and Gilman, A. G. (1996). Complexity and diversity of mammalian adenylyl cyclases. Annu. Rev. Pharmacol. Toxicol. 36, 461-480. doi: 10.1146/annurev.pa.36.040196.002333

Szklarczyk, D., Franceschini, A., Wyder, S., Forslund, K., Heller, D., Huerte-Cepas, J., et al. (2014). STRING v10: protein-protein interaction networks, integrated over the tree of life. Nucl. Acids Res. 43(Database issue): D447-D452. doi: 10.1093/nar/gku1003

Tarassishin, L., Suh, H.-S., and Lee, S. C. (2011). Interferon regulatory factor 3 plays an anti-inflammatory role in microglia by activating the PI3K/Akt pathway. J. Neuroinflamm. 8, 187-187. doi: 10.1186/1742-2094-8-187

Terada, L. S., and Nwariaku, F. E. (2011). Escaping Anoikis through ROS: ANGPTL4 controls integrin signaling through Nox1. Cancer Cell 19, 297-299. doi: 10.1016/j.ccr.2011.02.019

Terrien, E., Chaffotte, A., Lafage, M., Khan, Z., Préhaud, C., Cordier, F., et al. (2012). Interference with the PTEN-MAST2 interaction by a viral protein leads to cellular relocalization of PTEN. Sci. Signal. 5:ra58. doi: 10.1126/scisignal.2002941

Thanomsridetchai, N., Singhto, N., Tepsumethanon, V., Shuangshoti, S., Wacharapluesadee, S., Sinchaikul, S., et al. (2011). Comprehensive proteome analysis of hippocampus, brainstem, and spinal cord from paralytic and furious dogs naturally infected with rabies. J. Proteome Res. 10, 4911-4924. doi: $10.1021 / \mathrm{pr} 200276 \mathrm{u}$

Thoulouze, M. I., Lafage, M., Montano-Hirose, J. A., and Lafon, M. (1997). Rabies virus infects mouse and human lymphocytes and induces apoptosis. J. Virol. 71, $7372-7380$.

Thoulouze, M. I., Lafage, M., Yuste, V. J., Baloul, L., Edelman, L., Kroemer, G., et al. (2003a). High level of Bcl-2 counteracts apoptosis mediated by a live rabies virus vaccine strain and induces long-term infection. Virology 314, 549-561. doi: 10.1016/S0042-6822(03)00491-4

Thoulouze, M. I., Lafage, M., Yuste, V. J., Kroemer, G., Susin, S. A., Israel, N., et al. (2003b). Apoptosis inversely correlates with rabies virus neurotropism. Ann. N. Y. Acad. Sci. 1010, 598-603. doi: 10.1196/annals. 1299.112

Tomita, S., Nicoll, R. A., and Bredt, D. S. (2001). PDZ protein interactions regulating glutamate receptor function and plasticity. J. Cell Biol. 153, F19-F24. doi: $10.1083 /$ jcb.153.5.f19

Vaziri, B., Torkashvand, F., Eslami, N., and Fayaz, A. (2012). Comparative proteomics analysis of mice lymphocytes in early stages of infection by different strains of rabies virus. Indian J. Virol. 23, 311-316. doi: 10.1007/s13337-0120093-0

Venugopal, A. K., Ghantasala, S. S., Selvan, L. D., Mahadevan, A., Renuse, S., Kumar, P., et al. (2013). Quantitative proteomics for identifying biomarkers for Rabies. Clin. Proteomics 10:3. doi: 10.1186/1559-027 5-10-3

Vidy, A., Chelbi-Alix, M., and Blondel, D. (2005). Rabies virus P protein interacts with STAT1 and inhibits interferon signal transduction pathways. J. Virol. 79, 14411-14420. doi: 10.1128/JVI.79.22.14411-14420.2005

Wang, C.-Y., Lai, M.-D., Phan, N. N., Sun, Z., and Lin, Y.-C. (2015). Meta-analysis of public microarray datasets reveals voltage-gated calcium gene signatures in clinical cancer patients. PLOS ONE 10:e0125766. doi: 10.1371/journal.pone.0125766
Wang, X., Ning, Y., and Guo, X. (2015). Integrative meta-analysis of differentially expressed genes in osteoarthritis using microarray technology. Mol. Med. Rep. 12, 3439-3445. doi: 10.3892/mmr.2015.3790

Wang, X., Zhang, S., Sun, C., Yuan, Z. G., Wu, X., Wang, D., et al. (2011). Proteomic profiles of mouse neuro N2a cells infected with variant virulence of rabies viruses. J. Microbiol. Biotechnol. 21, 366-373.

Wang, Z. W., Sarmento, L., Wang, Y., Li, X. Q., Dhingra, V., Tseggai, T., et al. (2005). Attenuated rabies virus activates, while pathogenic rabies virus evades, the host innate immune responses in the central nervous system. J. Virol. 79, 12554-12565. doi: 10.1128/JVI.79.19.12554-12565.2005

Wong, N., and Wang, X. (2015). miRDB: an online resource for microRNA target prediction and functional annotations. Nucl. Acids Res. 43, D146-D152. doi: 10.1093/nar/gku1104

Yin, L., Coelho, S. G., Valencia, J. C., Ebsen, D., Mahns, A., Smuda, C., et al. (2015). Identification of genes expressed in hyperpigmented skin using metaanalysis of microarray datasets. J. Investig. Dermatol. 135, 2455-2463. doi: 10.1038/jid.2015.179

Yip, A. M., and Horvath, S. (2007). Gene network interconnectedness and the generalized topological overlap measure. BMC Bioinformatics 8:22. doi: 10.1186/1471-2105-8-22

Youdim, M. B. H. (2008). Brain iron deficiency and excess; cognitive impairment and neurodegeneration with involvement of striatum and hippocampus. Neurotoxicol. Res. 14, 45-56. doi: 10.1007/BF03033574

Yousaf, M. Z., Qasim, M., Zia, S., Khan, M. U. R., Ashfaq, U. A., and Khan, S. (2012). Rabies molecular virology, diagnosis, prevention and treatment. Virol. J. 9:50. doi: 10.1186/1743-422X-9-50

Zandi, F., Eslami, N., Soheili, M., Fayaz, A., Gholami, A., and Vaziri, B. (2009). Proteomics analysis of BHK-21 cells infected with a fixed strain of rabies virus. Proteomics 9, 2399-2407. doi: 10.1002/pmic.200701007

Zandi, F., Eslami, N., Torkashvand, F., Fayaz, A., Khalaj, V., and Vaziri, B. (2013). Expression changes of cytoskeletal associated proteins in proteomic profiling of neuroblastoma cells infected with different strains of rabies virus. J. Med. Virol. 85, 336-347. doi: 10.1002/jmv.23458

Zhao, P., Yang, Y., Feng, H., Zhao, L., Qin, J., Zhang, T., et al. (2013). Global gene expression changes in BV2 microglial cell line during rabies virus infection. Infect. Genet. Evol. 20, 257-269. doi: 10.1016/j.meegid.2013.09.016

Zhao, P., Zhao, L., Zhang, K., Feng, H., Wang, H., Wang, T., et al. (2012a). Infection with street strain rabies virus induces modulation of the microRNA profile of the mouse brain. Virol. J. 9, 159-159. doi: 10.1186/1743-422X-9-159

Zhao, P., Zhao, L., Zhang, T., Qi, Y., Wang, T., Liu, K., et al. (2011). Innate immune response gene expression profiles in central nervous system of mice infected with rabies virus. Comp. Immunol. Microbiol. Infect. Dis. 34, 503-512. doi: 10.1016/j.cimid.2011.09.003

Zhao, P., Zhao, L., Zhang, T., Wang, H., Qin, C., Yang, S., et al. (2012b). Changes in microRNA expression induced by rabies virus infection in mouse brains. Microb. Pathog. 52, 47-54. doi: 10.1016/j.micpath.2011.10.001

Ziff, E. B. (1997). Enlightening the postsynaptic density. Neuron 19, 1163-1174. doi: 10.1016/S0896-6273(00)80409-2

Conflict of Interest Statement: The authors declare that the research was conducted in the absence of any commercial or financial relationships that could be construed as a potential conflict of interest.

Copyright () 2016 Azimzadeh Jamalkandi, Mozhgani, Gholami Pourbadie, Mirzaie, Noorbakhsh, Vaziri, Gholami, Ansari-Pour and Jafari. This is an open-access article distributed under the terms of the Creative Commons Attribution License (CC BY). The use, distribution or reproduction in other forums is permitted, provided the original author(s) or licensor are credited and that the original publication in this journal is cited, in accordance with accepted academic practice. No use, distribution or reproduction is permitted which does not comply with these terms. 